Article

\title{
Multivariate DoE Optimization of Asymmetric Flow Field Flow Fractionation Coupled to Quantitative LC-MS/MS for Analysis of Lipoprotein Subclasses
}

\author{
Zsuzsanna Kuklenyik, Michael S. Gardner, Bryan A. Parks, David M. Schieltz, Jon C. Rees, \\ Lisa G. McWilliams, Yulanda M. Williamson, James L. Pirkle and John R. Barr * \\ Division of Laboratory Sciences, Centers for Disease Control and Prevention, National Center for \\ Environmental Health, Atlanta, GA 30341, USA \\ * Author to whom correspondence should be addressed; E-Mail: JBarr@cdc.gov. \\ Academic Editor: Gianni Galaverna
}

Received: 12 December 2014 / Accepted: 11 February 2015 / Published: 26 February 2015

\begin{abstract}
In this report we demonstrate a practical multivariate design of experiment (DoE) approach for asymmetric flow field-flow fractionation (AF4) method optimization using separation of lipoprotein subclasses as an example. First, with the aid of commercially available software, we built a full factorial screening design where the theoretical outcomes were calculated by applying established formulas that govern AF4 channel performance for a 5-35 $\mathrm{nm}$ particle size range of interest for lipid particles. Second, using the desirable ranges of instrumental parameters established from theoretical optimization, we performed fractional factorial DoE for AF4 separation of pure albumin and ferritin with UV detection to narrow the range of instrumental parameters and allow optimum size resolution while minimizing losses from membrane immobilization. Third, the optimal range of conditions were tested using response surface DoE for sub-fractionation of high and low density lipoproteins (HDL and LDL) in human serum, where the recovery of the analytes were monitored by fraction collection and isotope-dilution LC-MS/MS analysis of each individual fraction for cholesterol and apolipoproteins (ApoA-1 and ApoB-100). Our results show that DoE is an effective tool in combining AF4 theoretical knowledge and experimental data in finding the most optimal set of AF4 instrumental parameters for quantitative coupling with LC-MS/MS measurements.
\end{abstract}

Keywords: asymmetric flow field flow fractionation; mass spectrometry; lipoproteins; design of experiment; quality by design 


\section{Introduction}

Field-flow fractionation (FFF) is a group of separation techniques discovered in the 1970s by J. Calvin Giddings and coworkers [1]. In recent years, asymmetric flow FFF (AF4), specifically, has become a frequently used commercially available pre-analytical technique for measuring macromolecules, polymers and metal nanoparticles [2-5]. However, AF4 is generally still perceived as being difficult to optimize. In this report we demonstrate a design of experiment (DoE) workflow for optimization of AF4 methods. We adapted DoE (or quality-by-design, $\mathrm{QbD}$ ) approaches used by numerous software products (Drylab, ChromSwordAuto, ICOS, Osiris, Diamond, and PESOS), that are currently used for optimization of chromatographic separations. In this work, we demonstrate a similar approach, using a generic commercial statistical design software, JMP (SAS Institute). Applying DoE with a combination of the theoretical models and experimental data should help to make AF4 as an analytical separation technique more accessible to new users who want to develop and optimize their methods to specific analytes in the particle size range and resolution of interest.

An understanding of the AF4 theoretical principles is essential in implementation of AF4 applications (Table 1). The AF4 separation starts with sample injection (Figure 1a). While the sample is carried into the channel by the injection flow $\left(F_{i n j}\right)$, the focus flow $\left(F_{\text {focus }}\right)$ is also introduced into the AF4 channel opposite to the direction of $F_{i n j}$. With the simultaneous effect of the flow field created by the cross flow ( $\left.F_{\text {cross }}\right)$ through the molecular cut-off membrane covered frit wall, the analytes are focused near the membrane wall at a distance from the inlet where the longitudinal channel flow velocity reaches 0 , called the focusing point, $z$, which can be estimated by [6]

$$
\mathrm{z} \sim \frac{F_{\text {inj }}}{F_{\text {inj }}+F_{\text {focus }}} L_{f}
$$

where $L_{f}$ is the channel length between the $F_{i n j}$ inlet and $F_{f o c u s}$ inlet. Because of the used instrument design (Postnova) (Figure 1a), there is also a constant outlet flow, $F_{\text {out }}$ during injection. The user set flows are $F_{o u t}, F_{i n j}$ and $F_{c r o s s}$, while $F_{f o c u s}$ is automatically controlled as given by $F_{f o c u s}=F_{c r o s s}+F_{\text {out }}-F_{\text {inj }}$. The injection time, $t_{i n j}$, the time necessary for the transverse displacement of the analyte from the inlet to the accumulation wall, can be estimated by [6]

$$
t_{\text {inj }} \sim\left(\frac{V_{0}}{3 F_{\text {cross }}}\right)^{\frac{2}{3}}\left(\frac{w^{2}}{D_{i}}\right)^{\frac{1}{3}}
$$

where $D_{i}$ is the intrinsic diffusion coefficient of the analyte, $V_{0}$ is the channel volume, and $w$ is the channel thickness. For initial theoretical prediction $w$ can be treated simply as the thickness of the spacer inserted between the walls of the channel. However, due to swelling of the membrane, the actual channel thickness is usually changed by $\sim 10 \%$ of the spacer thickness which needs consideration when experimental data and theoretical predictions are compared. The instrumental parameters $w, t_{i n j}, F_{i n j}, F_{\text {cross }}$, and $F_{\text {out }}$, interactively control the injection/focusing process and have to be optimized to the $D_{i}$ of the analytes.

The injection/focusing step is followed by the AF4 elution (Figure $1 \mathrm{~b}$ ). After turning off $F_{\text {focus }}$, the inlet flow, $F_{\text {in }}$, is automatically controlled such that $F_{\text {in }}=F_{\text {cross }}+F_{\text {out }}$ throughout the entire AF4 run. The AF4 separation is based on the fundamental nature of laminar flow, where the laminar layers move slower near the walls of the channel than toward the middle of the channel, creating a parabolic flow velocity profile. As the individual particles begin to diffuse from the accumulation wall into the faster 
moving laminar layers toward the middle of the channel, the cross flow field simultaneously pulls them back closer to the accumulation wall. As a result, the molecules remain in $<10 \%$ of $w$ near the membrane wall throughout the separation process. Smaller molecules with larger $D_{i}$ 'leap' back and forth higher into faster moving laminar layers than larger molecules with smaller $D_{i}$, which result in a $D_{i}$ dependent elution order. By coupling the AF4 channel with a concentration detector (UV or RI) the separation of the analytes can be observed in the form of Gaussian peaks. The elution behavior of the analytes can be experimentally characterized by their peak retention level $R_{L}=t_{R} / t_{0}$, where $t_{R}$ is the retention time and $t_{0}$ is the void time. During their "leaping" along the wall, the molecules take up an average center of gravity distance $(l)$ from the wall. Expressing $l$ as a fraction of $w$ gives the retention parameter, $\lambda=l / w$. The retention parameter is correlated to the $D_{i}$ of the analytes by [6]

$$
D_{i}=\lambda \frac{w^{2} F_{\text {cross }}}{V_{0}}
$$

where $D_{i}$ is determined by the hydrodynamic diameter, $d_{h}$, according to the Stokes-Einstein Equation.

$$
D_{i}=\frac{k T}{3 \pi \eta d_{h}}
$$

\begin{tabular}{|c|c|c|c|c|c|}
\hline Symbol & Name & Unit & Symbol & Name & Unit \\
\hline$F_{i n i}$ & Injection flow & $\mathrm{mL} / \mathrm{min}$ & $z$ & Focusing point & $\mathrm{cm}$ \\
\hline$F_{\text {cross }}$ & Cross flow & $\mathrm{mL} / \mathrm{min}$ & $t_{0}$ & Void time & $\min$ \\
\hline$F_{\text {focus }}$ & Focus flow & $\mathrm{mL} / \mathrm{min}$ & $t_{R}$ & Retention time & $\min$ \\
\hline$F_{\text {out }}$ & Channel flow & $\mathrm{mL} / \mathrm{min}$ & $R_{L}$ & Retention level & \\
\hline$F_{\text {in }}$ & Inlet flow & $\mathrm{mL} / \mathrm{min}$ & $\lambda$ & Retention parameter & \\
\hline$F_{\text {det }}$ & Detector flow & $\mathrm{mL} / \mathrm{min}$ & $l$ & Center of gravity distance & $\mu \mathrm{m}$ \\
\hline$F_{\text {slot }}$ & Slot flow & $\mathrm{mL} / \mathrm{min}$ & $d_{h}$ & Hydrodynamic diameter & $\mathrm{nm}$ \\
\hline$F_{\text {cross }}^{\text {start }}$ & $\begin{array}{l}\text { Starting gradient cross } \\
\text { flow start }\end{array}$ & $\mathrm{mL} / \mathrm{min}$ & $D_{i}$ & Diffusion coefficient & $\mathrm{cm}^{2} / \mathrm{s}$ \\
\hline$F_{\text {cross }}$ end & $\begin{array}{l}\text { Ending gradient } \\
\text { cross flow }\end{array}$ & $\mathrm{mL} / \mathrm{min}$ & $w^{2} / D_{i}$ & Diffusion time across $w$ & $\min$ \\
\hline$t_{i n j}$ & Injection time & $\min$ & $W_{b t}$ & Peak width at peak base & $\min$ \\
\hline$t^{\text {end }}$ & $\begin{array}{l}\text { Cross flow gradient } \\
\text { run time }\end{array}$ & $\min$ & $W_{1 / 2}$ & $\begin{array}{l}\text { Peak width at half peak } \\
\text { height }\end{array}$ & $\min$ \\
\hline$n$ & $\begin{array}{l}\text { Gradient exponential } \\
\text { coefficient }\end{array}$ & & $H$ & Theoretical plate height & $\mu \mathrm{m}$ \\
\hline$L$ & Channel length & $\mathrm{cm}$ & $T$ & Temperature, 298 & K \\
\hline$L_{f}$ & $\begin{array}{l}\text { Distance of focus flow } \\
\text { inlet }\end{array}$ & $\mathrm{cm}$ & $k$ & $\begin{array}{l}\text { Boltzmann constant, } \\
1.38 \mathrm{e}-16\end{array}$ & $\mathrm{~g} \cdot \mathrm{cm}^{2} /\left(\mathrm{s}^{2} \cdot \mathrm{K}\right)$ \\
\hline$w$ & Channel thickness & $\mathrm{mm}$ & $\eta$ & Viscosity, 0.00894 & $\mathrm{~g} /(\mathrm{cm} \cdot \mathrm{s})$ \\
\hline$V_{0}$ & Channel volume & $\mathrm{mL}$ & & Cholesterol concentration & $\mathrm{mg} / \mathrm{dL}$ \\
\hline$V_{i n j}$ & Injection volume & $\mu \mathrm{L}$ & & Protein Concentration & $\mathrm{nmol} / \mathrm{L}(\mathrm{nM})$ \\
\hline
\end{tabular}

Table 1. Summary of symbols and units. 


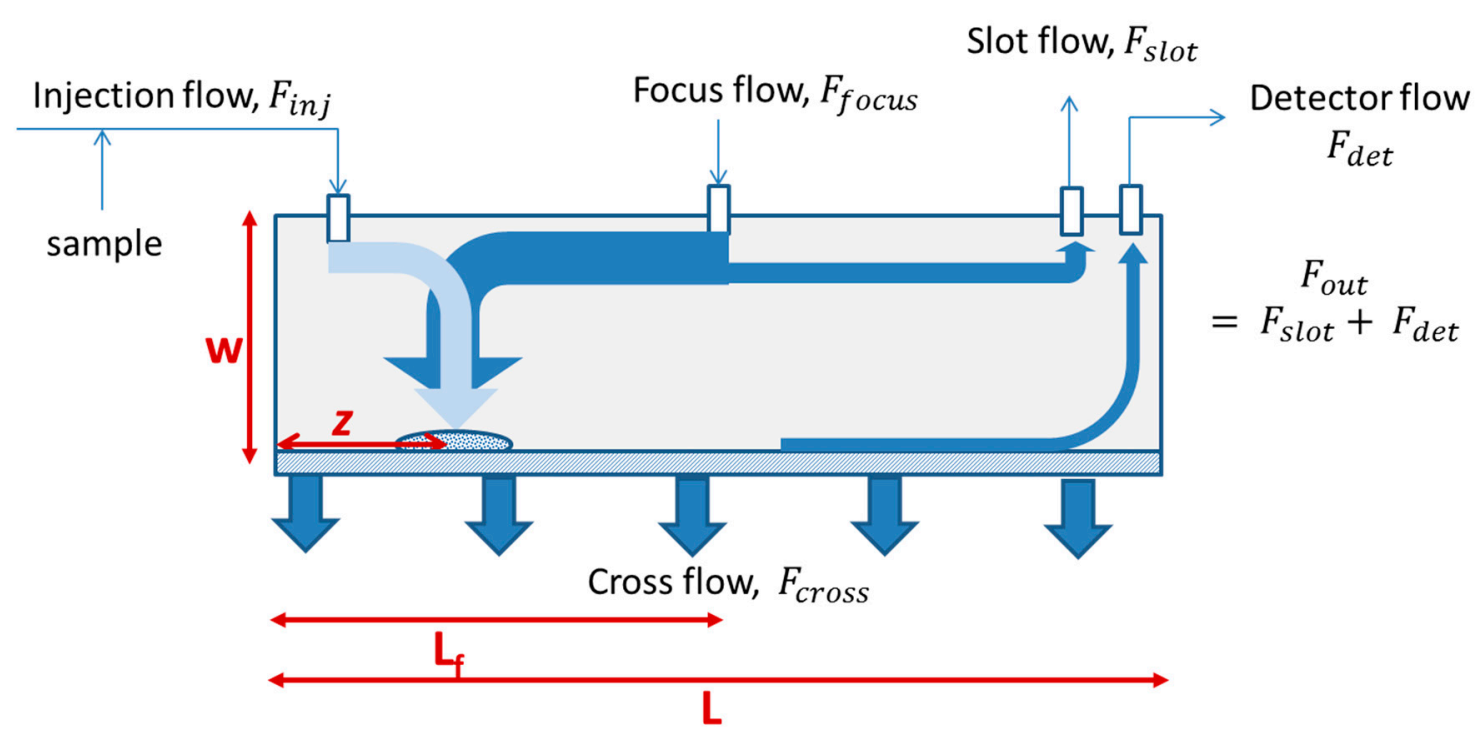

(A)

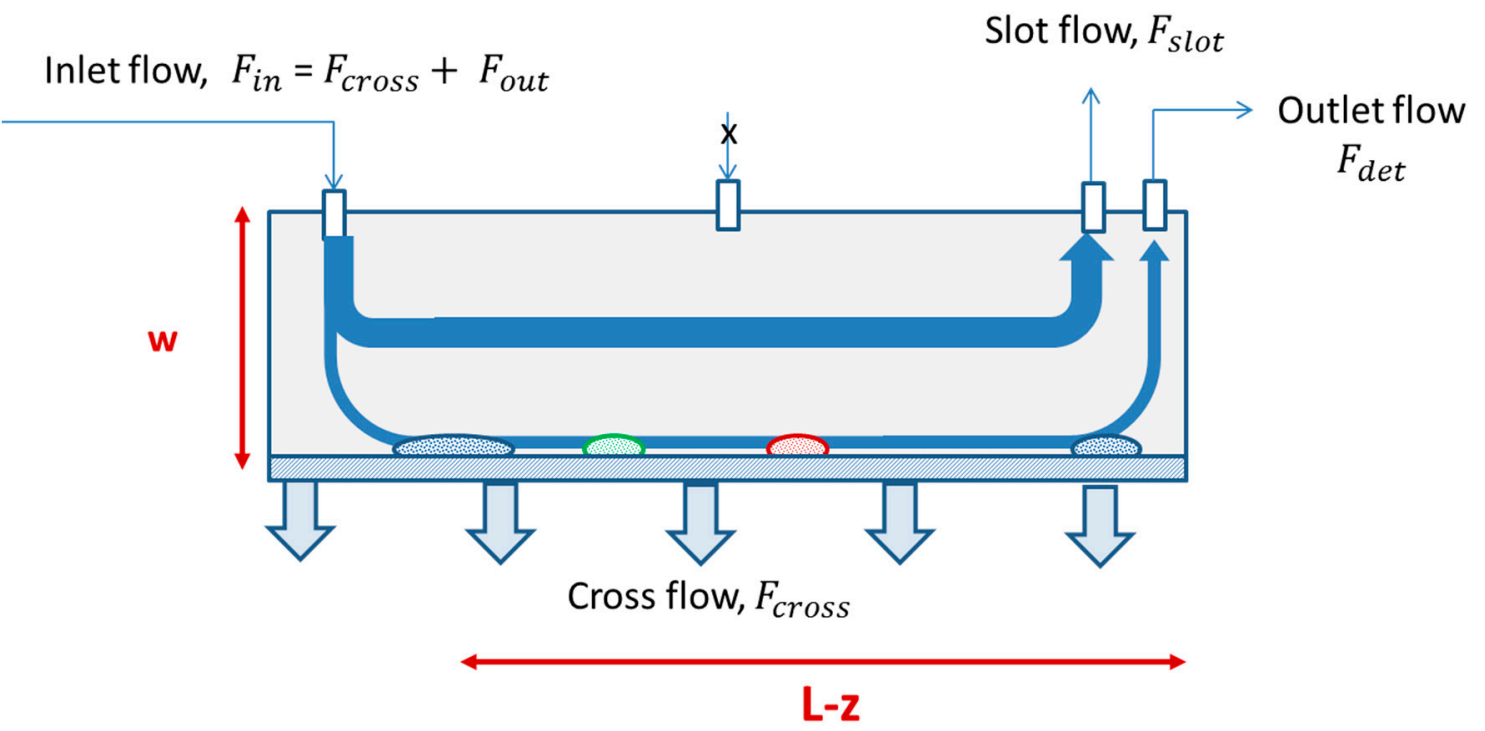

(B)

Figure 1. Schematic representation of the AF4 fluidic channel and adjustable flows during sample focusing (A) and fractionation (B). The injection flow $\left(F_{i n j}\right)$ delivers the injected sample from the autosampler loop into the inlet. Focusing flow $\left(F_{\text {focus }}\right)$ allows the steady state relaxation of the sample at the accumulation wall (molecular weight cut-off membrane). The cross flow $\left(F_{\text {cross }}\right)$ is pumped out through the accumulation wall. At the end of the channel, $F_{\text {out }}$ is split into a slot flow $\left(F_{\text {slot }}\right)$ which removes the analyte free upper laminar layers, and the detector flow $\left(F_{\text {det }}\right)$ which carries the analytes out of the channel to the detector and to the fraction collector. During the full course of the AF4 run $F_{\text {out }}=F_{\text {slot }}+F_{\text {det. }}$. During sample focusing, $F_{\text {focus }}$ is calculated from the other flows according to $F_{\text {focus }}=F_{\text {cross }}$ $+F_{\text {out }}-F_{\text {inj. }}$. During elution $F_{\text {in }}$ is automatically adjusted according to $F_{\text {in }}=F_{\text {out }}+F_{\text {cross }}$. 
Based on theoretical models, the observed $R_{L}$ can be predicted either from the theoretical parameter $\lambda$ or from the instrumental parameters, $w, V_{0}, F_{\text {cross }}$, and $F_{\text {out }}$ by [6]

$$
\begin{gathered}
R_{L}=\frac{t_{R}}{t_{0}}=\frac{1}{6 \lambda\left(\operatorname{coth}\left(\frac{1}{2 \lambda}\right)-2 \lambda\right)} \\
t_{0}=\frac{V_{0}}{F_{\text {cross }}} \ln \left(\frac{F_{\text {cross }}(L-z)+F_{\text {out }} L}{F_{\text {out }} L}\right) \\
t_{R}=\frac{w^{2}}{6 D_{i}} \ln \left(1+\frac{F_{\text {cross }}}{F_{\text {out }}}\right)
\end{gathered}
$$

These equations can be applied only with the condition that the retention level $R_{L}>3.3(\lambda<0.05)$, and the so called non-idealities are eliminated. These non-idealities are the interaction of the analytes with the molecular cut-off membrane which may cause retention time shifts (immobilization/remobilization delay), permanent adsorption which causes membrane fouling, and channel overloading which causes steric overloading effects apparent in peak asymmetry. With the above conditions at constant $F_{\text {cross }},<5 \%$ difference between theoretical and experimental AF4 retention times can be achieved [7].

The AF4 theoretical plate height $(H)$, the theoretical width of broadening for an analyte zone along the wall is estimated by $[8,9]$

$$
H=\frac{w^{2}}{9 D_{i}} \frac{1}{R_{L}^{3}} \frac{L-z}{t_{0}}
$$

from $H$ and $t_{R}$ the peak width at peak base $\left(\mathrm{W}_{b, t}\right)$ on the retention time scale can be calculated $[9,10]$

$$
W_{b, t}=\left(\frac{H}{(L-z)}\right)^{0.5} 4 t_{R}
$$

In order to decrease the run time and increase sample throughput, commercial instruments have the capability to use programmed cross flow, where the cross flow rate is programmed to decrease during the AF4 run according the exponential formula

$$
F_{\text {cross }}(\mathrm{t})=F_{\text {cross }}^{\text {start }}-\left(F_{\text {cross }}^{\text {start }}-F_{\text {cross }}^{\text {end }}\right)\left(\frac{t}{t^{\text {end }}}\right)^{n}
$$

The starting cross-flow $F_{\text {cross }}^{\text {start }}$ is usually equal to the $F_{\text {cross }}$ that is used during sample injection/focusing. The ending cross flow, $F_{\text {cross }}$ end, has to be high enough to maintain normal mode elution where Equations 4-9 can be applied. Throughout the gradient run a constant $F_{\text {out }}$ is maintained with automatic adjustment of $F_{\text {in }}$ according to $F_{\text {in }}=F_{\text {cross }}+F_{\text {out }}$.

All together, the AF4 method optimization involves the adjustment of nine instrumental parameters mentioned above ( $V_{i n j}, w, F_{i n j}, t_{i n j}, F_{\text {out }}, F_{\text {cross }}^{\text {start }}, t^{\text {end }}, F_{\text {cross }}$ end , and $\left.n\right)$. The suggested traditional approach is to start with a small $w$ and optimize $R_{L}$ to a 3-30 range by systematically varying $F_{\text {in }}, F_{\text {out }}$ and $F_{\text {cross }}$, without using a cross flow gradient program [7]. This is followed by the optimization of the focusing conditions, $F_{i n j}$ and $t_{i n j}$. Because of the complex inter-dependence of $F_{i n j}, t_{i n j}, F_{\text {out }}$ and $F_{\text {cross }}$, the user has to go back and forth between adjusting the focusing and elution conditions several times. Next, the injected sample amount $\left(V_{i n j}\right)$ is decreased until stable symmetrical fractogram peak shape and intensity is achieved. If the user wants to try a different channel spacer $(w)$, the whole optimization process has to be repeated. Finally, the cross flow gradient $\left(t^{\text {end }}, F_{\text {cross }}{ }^{\text {end }}\right.$, and $n$ ) is optimized to achieve a desired sample throughput.

The above optimization approach was designed with the principal purpose of using AF4 as a "first principle" hydrodynamic size measurement technique. However, in many practical applications AF4 is used as a pre-analytical technique where the molecule or particle size of the sample components is 
determined independently by other means, such as gradient gel electrophoresis, differential mobility analyzer (DMA) or electron microscopy. In these applications, the main purpose of using AF4 is to achieve size separation with optimal resolution to assess the relative amount of specific size components. Confirmation of size can be achieved by coupling AF4 with dynamic light scattering (DLS) or multi-angle light scattering (MALS) techniques, either on-line with a flow-through cuvette or off-line in collected fractions. An additional objective may be to collect size fractions followed by downstream quantitative chemical analysis with adequate sensitivity. In such pre-analytical applications the amount of sample injected into the AF4 channel has to be maximized while maintaining rugged size resolution, reasonable runtime, and reproducible maximum channel recovery (minimizing sample loss due to binding to the channel wall). Theoretical predictions of resolution can provide a good starting point; however achieving optimal channel recovery requires additional experiments.

Instead of a traditional AF4 optimization approach, we present here a multivariate DoE based approach using commercially available software. We use as an example the quantitative preparative separation of sub-classes of high and low density lipoproteins (HDL and LDL). The use of AF4 for the separation of HDL and LDL has been previously reported [11-18]. In this report we use HDL and LDL separation to demonstrate the DoE optimization approach. We show the quantitative ruggedness of the DoE optimized AF4 method with fraction collection and analysis of the individual fractions by means of stable isotope dilution liquid chromatography coupled with tandem mass spectrometry (LC-MS/MS) measurements.

\section{Materials and Methods}

\subsection{Reagents}

Phosphate buffered saline (PBS, $10 \mathrm{mM}$ sodium phosphate buffer/128 mM NaCl, $\mathrm{pH} 7.4$ ) was prepared from pre-mixed powder (Sigma-Aldrich, St. Louis, MO, USA) and deionized water. Bovine serum albumin (BSA), ferritin, potassium hydroxide, native cholesterol and stable isotope labeled cholesterol $\left(25,26,26,26,27,27,27-d_{7}\right)$ were purchased from Sigma-Aldrich (St. Louis, MO, USA). HPLC grade ethanol, isopropanol, methanol, hexanes, and nonane were purchased from Thermo-Fisher Scientific (USA). Calibration standard solution series of native cholesterol were prepared in $100 \%$ ethanol with a $0.141-704 \mu \mathrm{g} / \mathrm{mL}$ concentration range. The spiking solution of the isotope labeled cholesterol internal standard was $50 \mu \mathrm{g} / \mathrm{mL}$ in $100 \%$ ethanol. Native and ${ }^{13} \mathrm{C}$ labeled peptide calibration standards for bottom-up quantitative proteomic analysis were synthesized by MidWest BioTech Inc (Fishers, IN, USA). Peptides chosen for apolipoprotein A-1 (ApoA-1) were ATEHLSTLSEK (ATEHLST[L-U7]SEK) and DYVSQFEGSALGK (DYVSQFEGSA[L-U7]GK); peptides for apolipoproteinB-100 (ApoB-100) were ATGVLYDYVNK (ATGVLYDY[V-U6]NK) and ENFAGEATLQR (ENFAGEATL[Q-U7]R). The concentrations of the peptide stock solutions $(\sim 30 \mu \mathrm{g} / \mathrm{mL})$ were determined based on isotope dilution mass spectrometry based amino acid analysis with NIST certified amino acid calibrators [19]. Calibration standard series for ApoA-1 and ApoB-100 were prepared in $0.1 \%$ formic acid/water at $0.1-1000 \mathrm{pmol} / \mathrm{mL}$ concentrations. Lipoprotein depleted serum was obtained from Intracel Resources (Frederick, MD, USA), and serum samples from individual 
donors (QC1, QC2 and QC3) were purchased from Interstate Blood Bank (Saint Louis, MO, USA). The serum was distributed into $200-500 \mu \mathrm{L}$ aliquots immediately after shipment and stored frozen at $-80{ }^{\circ} \mathrm{C}$.

\subsection{Asymmetric Flow Field-Flow Fractionation (AF4)}

With injection of $20-80 \mu \mathrm{L}$ sample volumes, the separations were carried out on an AF2000 MultiFlow AF4 platform (Postnova Analytics, Salt Lake City, UT, USA). The AF4 system was equipped with an eluent degasser, tip flow pump $\left(F_{i n j}\right.$ and $\left.F_{i n}\right)$, focus flow pump $\left(F_{\text {focus }}\right)$, cross-flow syringe pump ( $F_{\text {cross }}$ ), slot pump (smart stream spitter) $\left(F_{\text {slot }}\right)$, autosampler (cooled to $5{ }^{\circ} \mathrm{C}$ temperature), and an integrated electronic interface unit. The carrier liquid was PBS buffer (pH 7.4). The membrane material was reconstructed cellulose. The standard Postnova kite shaped channel consisted of a large triangle at the inlet (base $/$ length $=3.5 \mathrm{~cm} / 2.0 \mathrm{~cm}$ ), a small triangle at the outlet (base $/$ length $=0.8 \mathrm{~cm} / 0.6 \mathrm{~cm}$ ) and a trapezoid in between. The total channel length was $28 \mathrm{~cm}$ with focus flow inlet at $17 \mathrm{~cm}$. Unless mentioned, all AF4 experiments were run with $0.2 \mathrm{~mL} / \mathrm{min}$ injection flow $\left(F_{i n j}\right)$, and $0.45 \mathrm{~mL} / \mathrm{min}$ outlet flow $\left(F_{\text {out }}\right)$, which was split into a $0.3 \mathrm{~mL} / \mathrm{min}$ slot flow $\left(F_{\text {slot }}\right)$ and a $0.15 \mathrm{~mL} / \mathrm{min}$ detector flow $\left(F_{\text {det }}\right)$. A $100 \mathrm{~cm}, 0.127 \mathrm{~mm}$ I.D. piece of peek tubing was placed between the $F_{\text {det }}$ outlet and the detector to maintain 6-8 bar channel backpressure. The cross flow $\left(F_{\text {cross }}\right)$ was $3.2 \mathrm{~mL} / \mathrm{min}$ during injection. After 1 min transition time, the cross-flow gradient started with $F_{\text {cross }}{ }^{\text {start }}=3.2 \mathrm{~mL} / \mathrm{min}$, followed by a $\left(\frac{t}{t^{\text {end }}}\right)^{n}$ exponential decay with $n=0.75$ and $\mathrm{t}^{\text {end }}=70 \mathrm{~min}$, ending with $F_{\text {cross }}$ end $=0.75 \mathrm{~mL} / \mathrm{min}$. The cross flow gradient elution was followed by $20 \mathrm{~min}$ purging at $2 \mathrm{~mL} / \mathrm{min}$ inlet flow with open purge valve. The $0.15 \mathrm{~mL} / \mathrm{min}$ detector flow was connected to a UV detector (Shimadzu) operated at $280 \mathrm{~nm}$ wavelength and an analytical fraction collector cooled to $5{ }^{\circ} \mathrm{C}$ temperature (Agilent Technologies). The delay volume between the UV detector and the fraction collector was $0.03 \mathrm{~mL}$. The fraction collection started with a 4-10 min delay after transition and lasted for 60-70 min with 1.5 or 2 min time increments for each fraction.

A typical AF4 batch of 8-16 injections (runs) resulted with collection into four 96-well Glass-Bottom Plates (Greiner Bio-One SensoPlate). The average hydrodynamic diameter $\left(d_{h}\right)$ in each fraction was measured by a Dynapro dynamic light scattering (DLS) plate reader (Wyatt Technologies, Santa Barbara, CA, USA) at $25{ }^{\circ} \mathrm{C}$. After size measurement, aliquots were transferred from the collection plates into separate 96 well plates using a Biomek FXP Laboratory Automation Workstation (BeckmanCouter Life Sciences, Brea, CA, USA); a $50 \mu \mathrm{L}$ aliquot was used for cholesterol analysis (in $0.5 \mathrm{~mL}$ polypropylene plate, round bottom, Agilent), and a $30 \mu \mathrm{L}$ aliquot for protein analysis (in $300 \mu \mathrm{L}$ Eppendorf PCR plate, Sigma-Aldrich, USA). The leftover fractions were spiked with $10 \mu \mathrm{L}$ of green food color (E143), and $50 \mu \mathrm{L}$ from each spiked well was transferred into an optical bottom 384-well plate (CYTOSTAR-T, Perkin-Elmer) and the volume of each fraction was calculated based on relative absorbance measured in a Spectramax 190 Tunable Microplate Reader (Conquer Scientific, USA).

\subsection{Cholesterol Analysis}

On the 96-well plates containing the $50 \mu \mathrm{L}$ fraction aliquots for cholesterol measurement, some of the empty wells were filled with $50 \mu \mathrm{L}$ of native cholesterol calibration standard (0.005-25 $\mu \mathrm{g}$ per well), and others with diluted serum (10 fold with PBS), for total serum cholesterol measurements. The plates (with AF4 fraction aliquots, calibration standards and diluted serum samples), were placed on the Beckman-Coulter Biomek FXP liquid handler deck, where each well was spiked with $10 \mu \mathrm{L}$ of isotopic 
labeled cholesterol internal standard (250 ng per well). To each well $0.2 \mathrm{~mL} 0.36 \mathrm{M} \mathrm{KOH}$ in $100 \%$ ethanol was added, followed by incubation at $60^{\circ} \mathrm{C}$ for $1 \mathrm{~h}$. After hydrolysis of the cholesterol esters, the reaction mix was neutralized by adding $115 \mu \mathrm{L}$ of $0.32 \mathrm{M} \mathrm{HCl}$. Next, the samples were evaporated to dryness with a Zipvap evaporator (Glas-Col, Terre Haute, IN, USA), and the free cholesterol was extracted by addition of $100 \mu \mathrm{L}$ of 1:1 nonane:isopropanol and mixed on a vortex mixer for $2 \mathrm{~min}$. The extracts were transferred into a $120-\mu \mathrm{L} 384$-well plate, which was covered with thermal-sealing foil and placed on the autosampler of the LC-MS/MS system. From the extracts $2 \mu \mathrm{L}$ was injected on a Luna HILIC $100 \mathrm{~mm} \times 2 \mathrm{~mm} 3 \mu \mathrm{m}$ HPLC column. The LC system was operated in gradient elution mode, with mobile phase A being hexanes, and mobile phase B composed of 90:10 ethanol:methanol. The gradient was as follows: $0 \%$ to $10 \%$ B over $2.0 \mathrm{~min}, 10 \%$ to $100 \%$ B over $1.0 \mathrm{~min}$, hold $100 \%$ B for $0.5 \mathrm{~min}$, return to $0 \% \mathrm{~B}$ over $0.1 \mathrm{~min}$, hold $1.4 \mathrm{~min}$. The flow rate was $0.6 \mathrm{~mL} / \mathrm{min}$. An AB Sciex 4000 Qtrap mass spectrometer was used with an atmospheric pressure chemical ionization probe (with nitrogen source gas for safety reasons) in positive ion mode (AB Sciex, Foster City, CA, USA). The native cholesterol and the isotopic labeled cholesterol internal standard chromatograms were acquired by multiple reaction monitoring (MRM), through $369 \rightarrow 161 \mathrm{~m} / \mathrm{z}$ and $376 \rightarrow 161 \mathrm{~m} / \mathrm{z}\left(\mathrm{d}_{7}\right)$, respectively. The precursor ions were produced by in-source water loss from the $(\mathrm{M}+\mathrm{H})^{+}$ions. The amount of cholesterol in each $50 \mu \mathrm{L}$ aliquot could be calculated from the native to internal standard peak area ratios. The limits of detection/quantification of cholesterol in the fractions were $0.4 / 1.2 \mu \mathrm{g} / \mathrm{mL}$, respectively. The amount of cholesterol in each fraction was calculated by [amount in the fraction] $=$ [concentration in the fraction]*[fraction volume]. Each fraction was treated as an individual sub-species of HDL and LDL in serum. This way we could estimate the limit of quantitation of the "cholesterol containing sub-species" in serum which was $0.1 \mathrm{mg} / \mathrm{dL}$. The measured amounts of "cholesterol containing sub-species" were converted into $\mathrm{mg} / \mathrm{dL}$ concentration in serum, by dividing with the serum volume injected into the AF4 system. Summing these individual sub-species concentrations in serum for 5-18 $\mathrm{nm}$ size range gave total $\mathrm{mg} / \mathrm{dL}$ concentration of HDL-cholesterol in serum, and summing for 18-30 nm size range gave total $\mathrm{mg} / \mathrm{dL}$ concentration of LDL-cholesterol in serum. The limit of quantitation for total HDL- and LDL-cholesterol in serum was $2 \mathrm{mg} / \mathrm{dL}$.

We also measured the total HDL cholesterol content of the serum samples directly without AF4 by precipitating LDL using $\alpha$-cyclodextrin/dextran sulfate/magnesium chloride reagent mix (Pointe Scientific, USA). After centrifugation, HDL cholesterol was measured in the supernatant with the same hydrolysis and LC-MS/MS procedure described above. These total HDL cholesterol concentrations were used for the calculation of the total HDL cholesterol injected into the AF4 channel. The channel recovery was calculated by the sum of the amounts of HDL sub-species found in the individual fractions divided by the amount of HDL cholesterol injected into the AF4 system.

\subsection{Apolipoprotein Analysis}

On the 96-well plates containing the $30 \mu \mathrm{L}$ fraction aliquots, empty wells were filled with $30 \mu \mathrm{L}$ of native peptide calibration standards ( $0.003-30 \mathrm{pmol}$ peptide per well), and triplicates of $30 \mu \mathrm{L}$ of 20 fold diluted serum for total serum protein measurements. The plates (with AF4 fraction aliquots, calibration standards and diluted serum samples), were placed on the Biomek FXP liquid handler, where to each well $3 \mu \mathrm{L} \mathrm{10 \%} \mathrm{Rapigest} \mathrm{detergent} \mathrm{(Waters} \mathrm{Life} \mathrm{Sciences,} \mathrm{USA)} \mathrm{solution,} \mathrm{and} 2 \mu \mathrm{L} 10 \mu \mathrm{g} / \mu \mathrm{L}$ trypsin 
(Promega, USA) were added. After incubation at $37^{\circ} \mathrm{C}$ for $1.5 \mathrm{~h}$, each well was spiked with $10 \mu \mathrm{L}$ of the isotopic labeled peptide internal standard mix (10 pmol/well), followed by an additional $1.5 \mathrm{~h}$ incubation at $37^{\circ} \mathrm{C}$. After trypsin digestion, $3 \mu \mathrm{L}$ of $2 \mathrm{M} \mathrm{HCl}$ was added to each well and the samples were incubated at $37{ }^{\circ} \mathrm{C}$ in order to degrade the Rapigest detergent. Samples were placed directly into the autosampler for LC-MS/MS analysis. The LC system (Acquity UPLC, Waters) was operated at $0.3 \mathrm{~mL} / \mathrm{min}$ flow rate. From the diluted digestion mix, $10 \mu \mathrm{L}$ was injected on a Mac-Mod HALO C18 core shell $100 \mathrm{~mm} \times 2.1 \mathrm{~mm}$ ID column (Mac-Mod Analytical). Solvents A and B were 100\% Water ( $0.1 \%$ Formic Acid) and Acetonitrile ( $0.1 \%$ formic acid). The gradient started at $2 \%$ solvent B for $0.5 \mathrm{~min}$. A linear gradient was applied to reach $95 \% \mathrm{~B}$ at $7 \mathrm{~min}$ and held for $0.5 \mathrm{~min}$. The column was then re-equilibrated to initial conditions at a flow rate of $0.6 \mathrm{~mL} / \mathrm{min}$ for a total runtime of $10 \mathrm{~min}$. An AB Sciex 6500 Qtrap mass spectrometer was used with heated electrospray ionization probe in positive ion mode (AB Sciex, Foster City, CA, USA). The native peptide and the isotopic labeled peptide internal standard chromatograms were acquired by multiple reaction monitoring. The amount of peptide in each $30 \mu \mathrm{L}$ aliquot could be calculated from the native to internal standard peak area ratios. The limits of detection/quantification of peptides in the fractions were $0.4 / 1.2 \mathrm{pmol} / \mathrm{mL}$. The amounts of peptides in each fraction was calculated by [amount in the fraction] $=$ [concentration in the fraction] $*$ [fraction volume]. The molar amounts of peptide gave the molar amounts of protein (ApoA-I or ApoB-100) in the fractions. Each fraction was treated as an individual sub-species of the protein analyte in serum. The measured molar amounts of "protein containing sub-species" were converted into $\mathrm{nmol} / \mathrm{L}$ (or $\mathrm{nM}$ ) concentration in serum, by dividing with the serum volume injected into the AF4 system: [protein concentration in the fraction]*[fraction volume]/[volume of serum injected serum]. This way we could estimate the limit of quantitation of the "protein containing sub-species" in serum which was $8 \mathrm{nmol} / \mathrm{L}$. Summing these individual "ApoA-I containing sub-species" concentrations in serum for 5-18 nm size range gave total $\mathrm{nmol} / \mathrm{L}$ concentration of HDL-ApoA-I in serum, and summing the "ApoB-100 containing sub-species" concentration for 18-30 nm size range gave total LDL-ApoB-100 concentration in serum. The limit of quantitation of the total HDL-ApoA-I and LDL-ApoB-100 in serum was $\sim 20 \mathrm{nmol} / \mathrm{L}$.

\subsection{Design of Experiment (DOE) and Modelling}

DoE tables were created using JMP Statistical Discovery software (SAS Institute Inc., Cary, NC, USA). The experiments were evaluated using least squares fit modeling with incorporation of both individual factors $\left(\mathrm{X}_{1}, \mathrm{X}_{2}, \mathrm{X}_{3}, \ldots, \mathrm{X}_{1}^{2}, \mathrm{X}_{2}^{2}, \mathrm{X}_{3}^{2}, \ldots\right)$, and second order and third order cross interaction terms $\left(\mathrm{X}_{1} \mathrm{X}_{2}, \mathrm{X}_{1} \mathrm{X}_{3}, \mathrm{X}_{2} \mathrm{X}_{3}, \ldots\right.$ and $\left.\mathrm{X}_{1} \mathrm{X}_{2} \mathrm{X}_{3}\right)$. The complex multidimensional models could be viewed in the form of prediction profiles (two dimensional slices) for each outcome $v s$. individual factor. Each prediction trace represented the predicted response as one variable is changed while the others are held constant at the selected values. The polynomial model predicted individual outcomes were weighted with desirability functions on a scale of $0-1$, expressing the need for a preferred maximum, minimum or target values. The optimal set of instrument parameters was determined from the maxima of the combined desirability for each factor which was calculated by incorporating the desirability of all outcomes. The outcomes were also visualized in JMP by two dimensional contour plots as a function of different pair combinations of $w, w^{2} / D_{i}, F_{\text {cross }} / F_{\text {out }}$, and $F_{\text {inj }} / F_{\text {cross. }}$. 


\section{Results and Discussion}

\subsection{Theoretical Optimization}

To better understand the complex interaction of the different instrument parameters, first we performed a theoretical (simulated) DoE experiment. Using the DoE menu in the JMP software, we generated a 7 factor $/ 5$ level full factorial design table with all possible combinations of $d_{h}, w, F_{\text {cross }}, F_{\text {out }}$, $F_{i n j}, n$, and $t^{\text {end }}$, sampling all possible interaction terms $\left(\mathrm{X}_{1} \mathrm{X}_{2}, \mathrm{X}_{1} \mathrm{X}_{3}, \mathrm{X}_{2} \mathrm{X}_{3}, .\right.$. etc. $)$ with $F_{\text {cross }}=F_{\text {cross }}^{\text {start }}$, and $F_{\text {cross }}{ }^{\text {end }}=0$. We created the design table for $d_{h}=5-35 \mathrm{~nm}$. We only sampled $F_{\text {cross }} \geq F_{\text {out }}$ values. This full factorial DoE design table had 78128 rows (theoretical runs). We calculated theoretical outcomes by applying formulas 1-10 (see Introduction) for each 76128 rows, including $D_{i}$, $\lambda, l, H, z, t_{i n j}, t_{0}, t_{R}, W_{b, t}$, and $R_{L}$, both at $F_{c r o s s}{ }^{\text {start }}$ and at $F_{c r o s s}{ }^{t / 2}$ at the $1 / 2$ time point of the cross flow gradient run time with $F_{\text {cross }}$ end $=0$.

Using the theoretical outcomes, we generated a least squares fit multidimensional polynomial model using $d_{h}, w, F_{\text {cross }}, F_{\text {out }}, F_{i n j}, n$, and $t^{\text {end }}$, as x variables. We also calculated a response surface least squares fit model using factor attributes $w^{2} / D_{i}, F_{\text {cross }} / F_{\text {out }}$, and $F_{i n j} / F_{\text {cross }}$ as x variables. Statistical $\mathrm{R}^{2}$ were in the 0.90-0.99 range for both models. The JMP statistical design software allowed for viewing model predictions in multiple ways. One we found very useful was the so called desirability optimization program interface, which allowed interactive optimization of the most desirable ranges of instrumental parameters to a selected particle size (see Section 2.5 and Figure S1). The visually most assessable way of showing the results was the contour profiles. For diameters 7 and $30 \mathrm{~nm}, t_{R}, W_{b, t,} z$ and $t_{i n j}$ contour profiles are shown in Figure 2A,C and 2B,D, respectively. Based on graphs 2A and $2 \mathrm{~B}$ at different $F_{i n j} / F_{\text {cross }}$ ratios, the $t_{i n j}$ and $z$ can be determined for a specific $w$. From graphs $2 \mathrm{C}$ and $2 \mathrm{D}$ at different $F_{\text {cross }} / F_{\text {out }}$ ratios, the desired $t_{R}$ and $W_{b, t}$, can be determined. These graphs reflect Equations $1-8$, showing visually how $t_{R}$ and $t_{i n j}$ is size dependent, while $W_{b, t}$ and $z$ is not. From the $F_{\text {inj }} / F_{\text {cross }}$ and $F_{\text {cross }} / F_{\text {out }}$ ratios a workable range of $F_{\text {inj }}, F_{\text {cross }}$ and $F_{\text {out }}$ can be estimated.

More generic contour profiles for the entire 5-35 $\mathrm{nm}$ range (Figure 3) were created by introducing $w^{2} / D_{i}$ as an x-variable into our model. The term $w^{2} / D_{i}$ has a physical connotation, meaning the diffusion time for different size molecules across a different length of $w$. First $w^{2} / D_{i}$ can be determined from Figure $3 \mathrm{~A}$ based on $d_{h}$ and $w$. Using the $w^{2} / D_{i}$ values, the $F_{\text {cross }} / F_{\text {out }}$ ratio can be determined for a desired $t_{R}$ and corresponding $W_{b, t}$ from Figure 3B, and for desired $t_{i n j}$ and corresponding $z$ from Figure 3C. The contour profiles for $R_{L}$ and $l$ in Figure 3D shows that high $F_{\text {cross }} / F_{\text {out }}$ ratio leads high $R_{L}$ and very low $l$ (center of gravity distance from the membrane) which increases the likelihood of particle-membrane interactions and non-ideal elution behaviors. Using the prediction profiles and contour plots allowed us to narrow the range of AF4 parameters to the specific size range of interest before the actual laboratory experiments. In essence, these contour plots and prediction profiles provided a quick and easily accessible visual overview of the complex AF4 parameter relationships that are described in Equations 1-10, and highlighted in numerous publications [6-8,20,21]. We generated these graphs for 5-35 nm diameter range, but the 78128 row table can be easily modified to any size range and relevant $w^{2} / D_{i}$ values and $F_{\text {cross }} / F_{\text {out }}$ ratios (see example for $30-350 \mathrm{~nm}$ in supplementary Figures S2 and S3). 

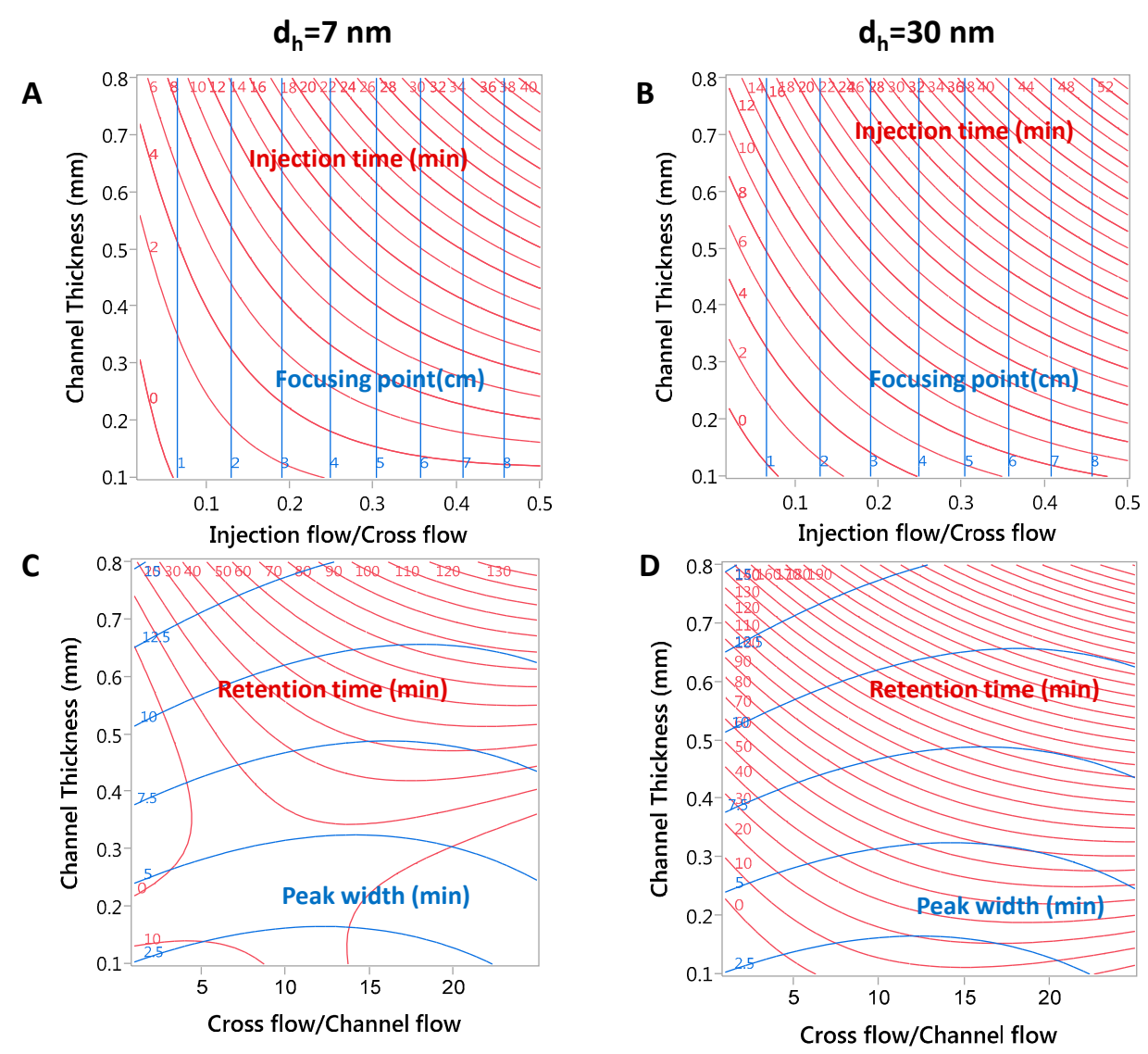

Figure 2. Contour profiles for $7 \mathrm{~nm}$ and $30 \mathrm{~nm}$ diameter particles: overlay of injection time $\left(t_{i n j}\right)$ and focusing point $(z)$ as a function of channel thickness $(w)$ and injection flow/cross flow ratio $\left(F_{\text {inj }} / F_{\text {cross }}\right)$ (A and $\left.\mathbf{B}\right)$, and theoretical retention time $\left(t_{R}\right)$ and peak width $\left(W_{b, t}\right)$ as a function of channel thickness $(w)$ and cross flow/channel flow ratio $\left(F_{\text {cross }} / F_{\text {out }}\right)(\mathbf{C}$ and $\mathbf{D})$, respectively. Based on graphs A and B the $t_{i n j}$ can be determined for a specific $w\left(F_{i n j}=0.3 \mathrm{~mL} / \mathrm{min}\right.$ and $\left.F_{\text {cross }} / F_{\text {out }}=7\right)$. From graphs $\mathrm{C}$ and $\mathrm{D}$ the $F_{\text {cross }} / F_{\text {out }}$ ratio can be determined for a desired $t_{R}$ for 7 and $30 \mathrm{~nm}$ particles $\left(F_{\text {inj }} / F_{\text {cross }}=0.1\right)$.

\subsection{DoE Optimization with Protein Standards}

To test the accuracy of our theoretical model based predictions experimentally, we generated a 7 factor fractional factorial design table with 54 runs incorporating second and third order cross effects $\left(\mathrm{X}_{1} \mathrm{X}_{2}\right.$ and $\mathrm{X}_{1} \mathrm{X}_{2} \mathrm{X}_{3}$ ) (Supplementary Table S1). The ranges of the AF4 parameters were $F_{\text {cross }}=1-5 \mathrm{~mL} / \mathrm{min}$, $F_{\text {out }}=0.25-1 \mathrm{~mL} / \mathrm{min}, t_{i n j}=1-10 \mathrm{~min}$ and $n=0.6-1$. We injected the same mix of $2 \mathrm{mg} / \mathrm{mL} \mathrm{BSA}$ and $2 \mathrm{mg} / \mathrm{mL}$ ferritin with varied injection volumes $V_{i n j}=20-80 \mu \mathrm{L}$. We chose channel spacers with $0.19,0.35$ and $0.5 \mathrm{~mm}$ thickness. We completed these runs in three unattended overnight batches, changing the channel spacer in between. For all $54 \mathrm{runs}$, we used $F_{i n j}=0.2 \mathrm{~mL} / \mathrm{min}, t_{\text {run }}=80 \mathrm{~min}$, and $F_{\text {cross }}{ }^{\text {end }}=0 \mathrm{~mL} / \mathrm{min}$. After completing the experiments, we measured the BSA and ferritin $t_{R}$, BSA-ferritin $W_{l / 2}$ (peak width at half peak height), and UV peak areas in each fractogram. We calculated the channel thickness from the measured BSA retention time to correct for the swelling of the membrane. With channel spacers $0.19,0.35$, and $0.50 \mathrm{~mm}$ the calculated channel thickness was $0.17,0.32$, and $0.43 \mathrm{~mm}$, respectively, with $10 \%-11 \% \mathrm{CV}$ for all 54 injections of the DOE experiment. We calculated the BSA-ferritin peak resolution $\operatorname{Res}=\Delta t_{R} / \bar{W}_{1 / 2}$, and the percent relative channel recovery as the sum of the 
peak areas divided by the injection volume $\left(V_{i n j}\right)$ and normalized for the maximum value of all 54 runs. The correction of the signal areas for different $F_{\text {out }}$ was not necessary because we used "slotting" (Figure 1), permitting the split of the outlet flow $\left(F_{\text {out }}=F_{\text {det }}+F_{\text {slot }}\right)$ into a constant analyte containing detector flow $\left(F_{d e t}\right)$ and varied the slot flow $\left(F_{\text {slot }}\right)$ that contained buffer only ( $<1 \%$ loss of analytes). We also calculated the theoretical $t_{R}$ for ferritin and the theoretical BSA-ferritin resolution $\left(R e s=\Delta \mathrm{t}_{\mathrm{R}} / \bar{W}_{b, t}\right)$ using corrected channel thickness values. The correlation slope between experimental and theoretical values was 0.99 $\left(\mathrm{R}^{2}-=0.92\right)$ for the ferritin $t_{R}$ and $0.48\left(\mathrm{R}^{2}-=0.75\right)$ for the BSA-ferritin resolution (Figure $\left.\mathrm{S} 4\right)$.
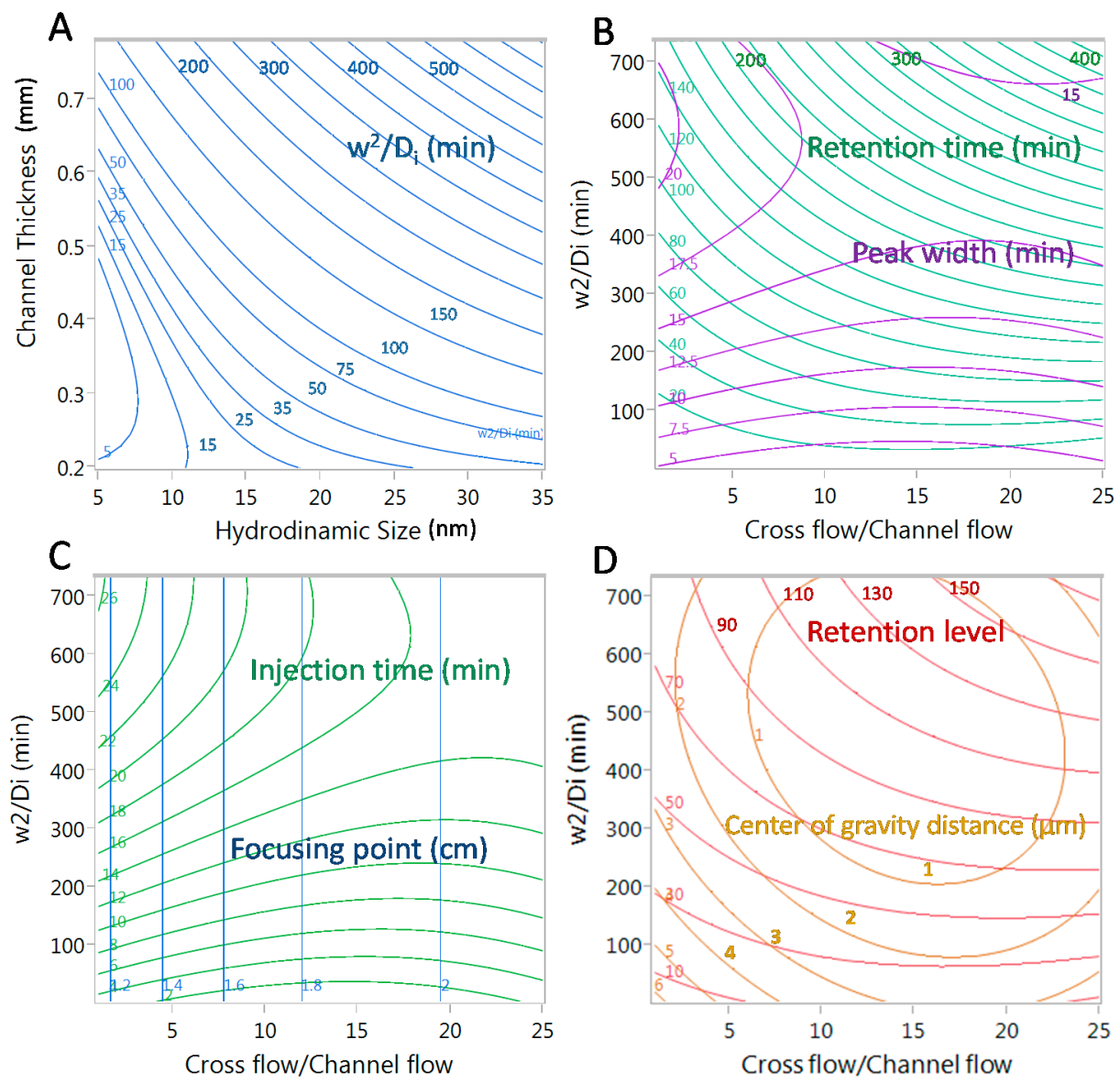

Figure 3. Theoretical contour profiles for $5-35 \mathrm{~nm}$ size range at $F_{i n j} / F_{\text {cross }}=0.1$. First $w^{2} / D_{i}$, can be determined from Figure $\mathbf{A}$ based on hydrodynamic diameter and the channel thickness. Using the $w^{2} / D_{i}$ values, the cross flow/channel flow ratio $\left(F_{\text {cross }} / F_{\text {out }}\right)$ can be determined for a desired retention time $\left(t_{R}\right)$ and corresponding peak width $\left(W_{b, t}\right)$ from Figure B. With the selected $F_{\text {cross }} / F_{\text {out }}$ ratio the injection time $\left(t_{i n j}\right)$ and corresponding focusing point $(z)$ is determined from Figure $\mathbf{C}$. The corresponding retention level $\left(R_{L}\right)$ and center of gravity distance from the membrane $(l)$ can be determined from Figure $\mathbf{D}$.

There were statistically significant trends $(\mathrm{p}<0.05)$ in resolution and channel recovery as a function of increasing $w$ and $F_{\text {cross }} / F_{\text {out }}$ ratio by the response surface least squares fit model generated based on the experimental data (Figure 4 and supplementary Figures S5 and S6). The empirical contour profiles show that resolution $\operatorname{Res}=\Delta t_{R} / \bar{W}_{1 / 2}$ is strongly affected by $w$ (Figure $4 \mathrm{~A}$ ). Increasing $w$ "magnifies" the effect of the $F_{\text {cross }} / F_{\text {out }}$ ratio on resolution; in accordance with Equations 5-9, $\Delta t_{R} \sim w^{2}$ while $W_{b, t}$ is independent of $w$. While resolution increases with greater $w$ and $F_{\text {cross }} / F_{\text {out }}$ ratio, recovery goes through 
a maximum (Figure 4B). The calculated center of gravity distance from the membrane at maximum recovery was 2.5-3 $\mu \mathrm{m}$ (Figure $4 \mathrm{C}$ ) and the optimal injection time was 8-10 min (Figure 4D). The maximum relative recovery predicted by the empirical model was only $65 \%$. A possible explanation for this recovery is that we used only UV detection where at extreme conditions (lowest $w$ and $F_{\text {cross }} / F_{\text {out }}$ ratios) the BSA and ferritin peaks (and possible other aggregates) were all in overlap. This may lead to overestimation of the highest recovery which was used for the calculation of the relative recoveries. Furthermore, several parameters were changed during the experiments, including injection volume and gradient exponential. Although we divided all UV peak areas with the injection volume, the ranges of other parameters were still quite wide. The program statistically fits all data points with one multivariate model which may underestimate the recovery at the optimum point. Nevertheless, these contour profiles demonstrate that the range of AF4 experimental conditions where sufficient resolution and recovery can be achieved simultaneously is fairly narrow and requires the consideration of multiple parameters. Without effectively sampling the AF4 parameter space by DoE, understanding and accounting for all the complex cross effects among the instrumental parameters would have been much more difficult.

A

Experimental Resolution
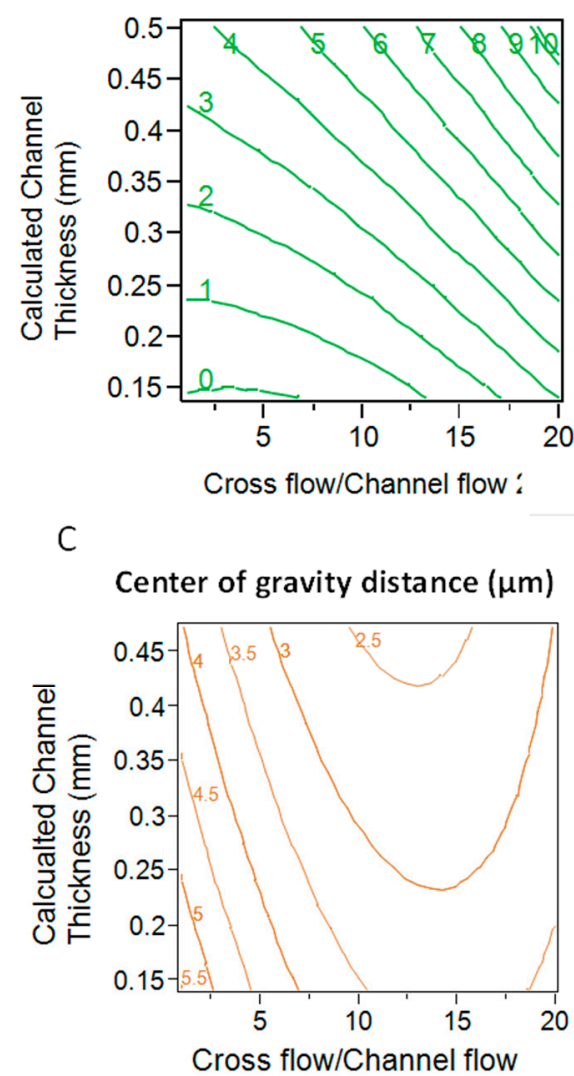

B

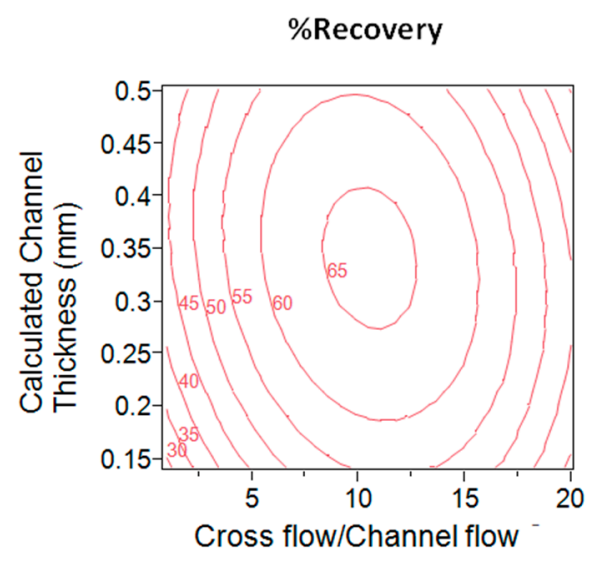

D

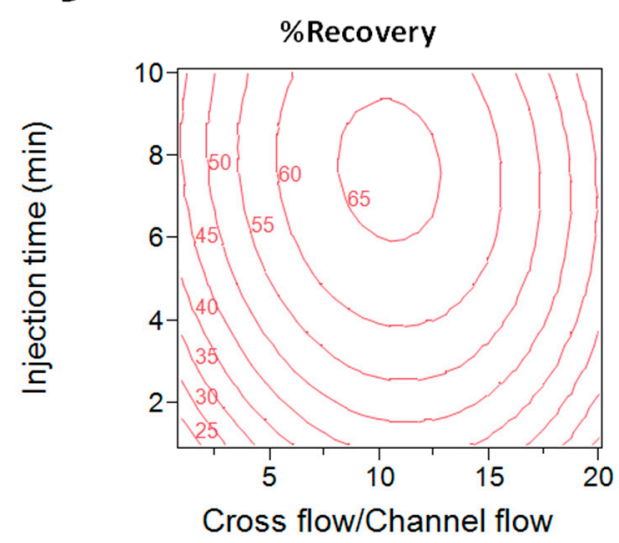

Figure 4. Contour profile representation of the experimental data based response surface least square fit model for bovine serum albumin and ferritin standards. Root mean square error for resolution $\left(\Delta t_{R} / \bar{W}_{1 / 2}\right)$ was \pm 0.7 , for \%recovery it was $\pm 13 \%$, and for the center of gravity distance it was $\pm 0.4 \mu \mathrm{m}$. For the calculation of each contour plot other parameters were constant: $t_{i n j}=8 \mathrm{~min}(\mathbf{A}, \mathbf{B}, \mathbf{C}), F_{i n j} / F_{c r o s s}=0.06(\mathbf{A}-\mathbf{D}), V_{i n j}=80 \mu \mathrm{L}$. (A-D): Corresponding fractograms are shown in Supplementary Figure S3. 
Each graph in Figure 4 shows the interaction between only two parameters in the model while other parameters were set constant as indicated in the figure legend. The optimal parameters were elucidated by considering all parameters at the same time, for which we used JMP software functions. The graphical output of the software is shown in supplementary material (Figure S4). This output was obtained with the so called desirability optimization function of the JMP software which is described in Section 2.5 and in Figure S4.

Maintaining linear relationship between $d_{h}$ and $t_{R}$ was also our priority. For confirmation, we selected fractograms collected with 0.35 and $0.5 \mathrm{~mm}$ spacer thickness, and plotted the $t_{R}$ of the monomers and dimers of BSA and ferritin against their expected $d_{h}$ (Figure 5). The linearity of the plots is evidence that the DoE data based range of optimal conditions resulted in normal mode elution, where $d_{h}$ and $t_{R}$ are in an approximate linear relationship.

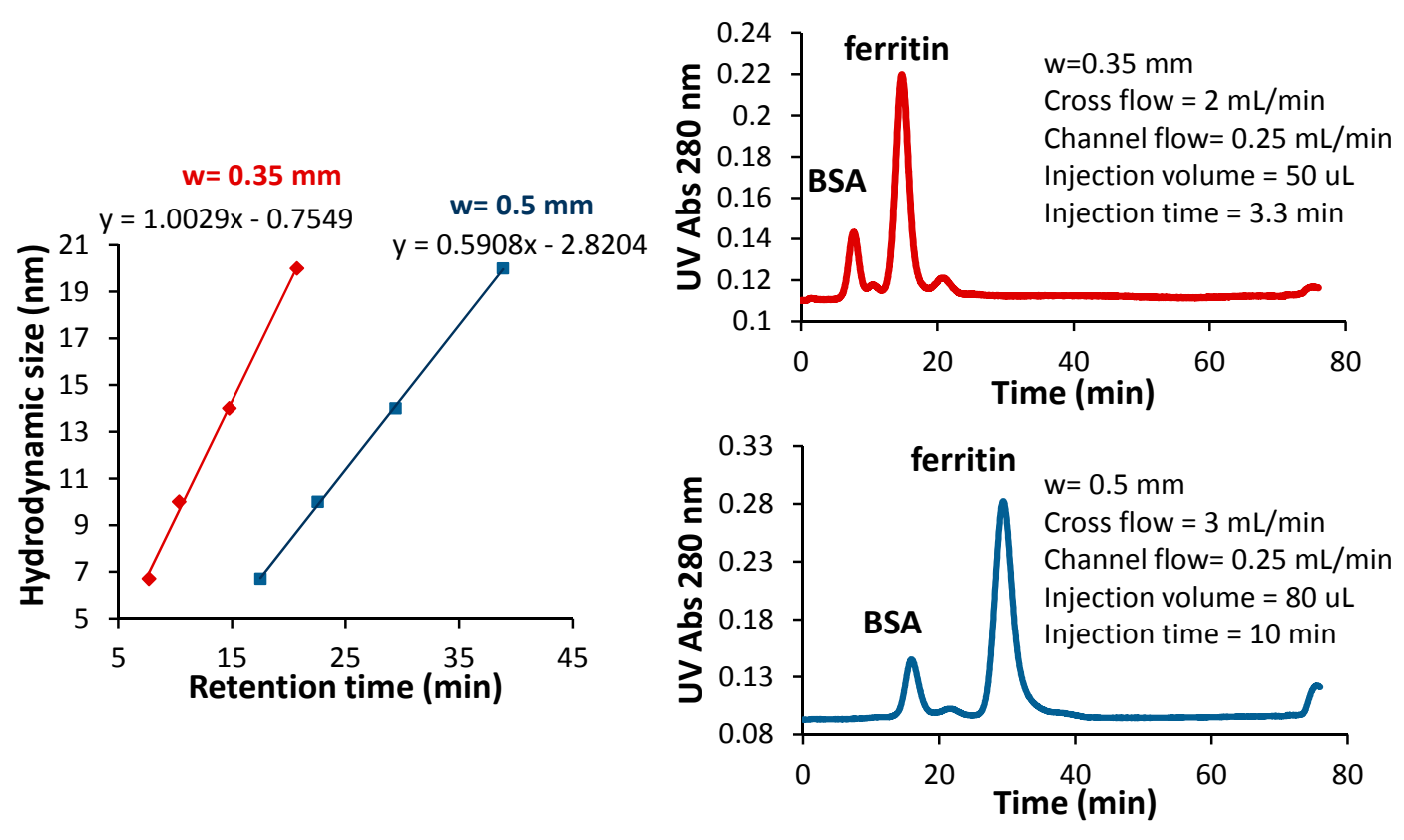

Figure 5. Retention times of bovine serum albumin (BSA) and ferritin monomers and dimers plotted against their expected hydrodynamic size, confirmed by dynamic light scattering measurements in corresponding collected fractions (right).

\subsection{DoE Optimization with Human Serum}

Based on the above theoretical and experimental DoE evaluation, we were able to set several experimental variables $\left(F_{i n j}=0.2 \mathrm{~mL} / \mathrm{min}, n=0.75, t_{\text {run }}=70 \mathrm{~min}, F_{\text {cross }}\right.$ end $=0.75 \mathrm{~mL} / \mathrm{min}$ and channel spacer $w=0.5 \mathrm{~mm}$ ). We turned to our analytes and matrix of interest, high and low density lipoproteins (HDL and LDL) in human serum. We built a 3-factor response surface design varying only the $t_{\text {inj }}=3-7 \mathrm{~min}, F_{\text {cross }}=3-4.5 \mathrm{~mL} / \mathrm{min}$ and $F_{\text {out }}=0.45-0.8 \mathrm{~mL} / \mathrm{min}$. This led to a 15 run-experiment (Supplementary Table S2) which we performed in one overnight batch sequence with injecting $80 \mu \mathrm{L}$ of the same serum sample (QC1). The fractograms had three prominent UV absorbance peaks, containing mainly human serum albumin (HSA), HDL and LDL. The resolution was calculated from the HDL-LDL $\Delta t_{R}$ (measured from cholesterol profiles) divided by the $\mathrm{W}_{1 / 2}$ of HSA, being the most prominent peak in the UV fractogram. Recovery was monitored by the sum of the LDL and HDL 
cholesterol content of the fractions. The UV peak area during the purge was also monitored. Representative fractograms can be seen in Supplementary Figure S6.

Building a least squares fit response surface model based on the results of the 15 runs (Figure 6), the desirability optimization revealed the final set of parameters: $F_{\text {cross }}=3.2 \mathrm{~mL} / \mathrm{min}, F_{\text {out }}=0.45 \mathrm{~mL} / \mathrm{min}$ and $t_{i n j}=7 \mathrm{~min}$. The predicted $l=w^{2} /\left(6 R_{L}\right)$ for the LDL peak at these conditions was $5 \mu \mathrm{m}$.

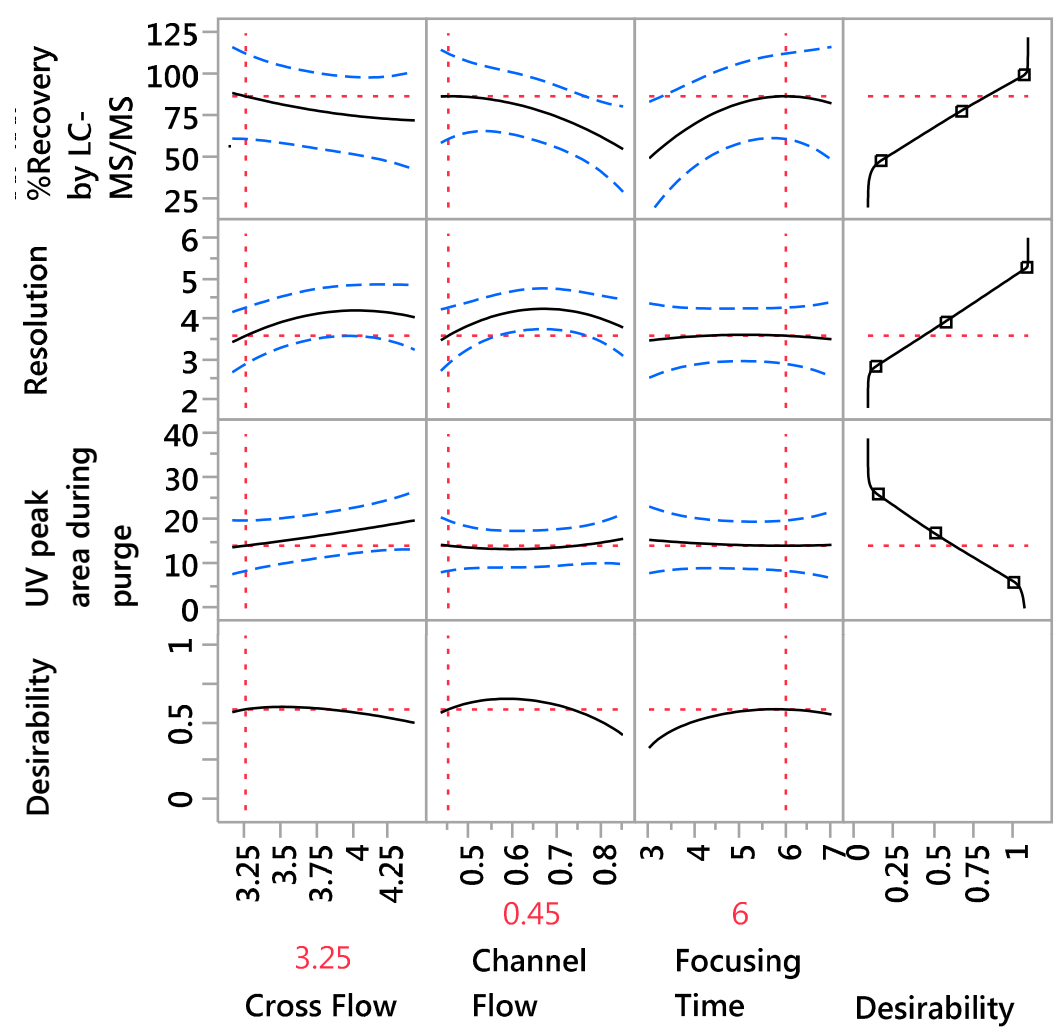

Figure 6. Desirability optimization of AF4 instrumental parameters for separation of high density and low density lipoprotein (HDL and LDL) sub-classes in human serum samples. Each prediction profile (continuous line) is a predicted response as one variable is changed while the others are held constant at selected values (vertical red lines). The prediction profiles are t-tested against true value zero (horizontal red dotted line) with probability criteria of p-value $<0.05$ (dashed lines around solid line). Intersection between horizontal red lines and blue probability criteria lines indicate statistically significant prediction profiles. The desirably function of each outcome (far right column), was combined with the prediction profiles resulting in combined desirability function for each AF4 parameter (bottom row), which was used to determine the optimal set of parameters.

To test the linearity of the channel recovery at the above optimal conditions, we prepared a dilution series from a relatively high HDL containing sample (QC3) with lipoprotein depleted serum. We analyzed each fraction for cholesterol and calculated the sum of the HDL fractions expressed in $\mathrm{mg} / \mathrm{dL}$ in serum. The dilution series gave a linear response with $\mathrm{R}^{2}=0.968$ (Figure 7A) and stable retention times (Figure 7B). We note that the lipoprotein depleted serum had a minimum amount of lipoproteins but did have other proteins at relatively high concentration. The overlapping peaks from 10-30 min are from these other proteins in the depleted serum plus the added HDL/proteins from the non-depleted 
serum (QC3). As the contribution of the depleted serum decreased while the non-depleted serum components increased, the maxima of the merged UV peaks at 10-30 min shifted somewhat.
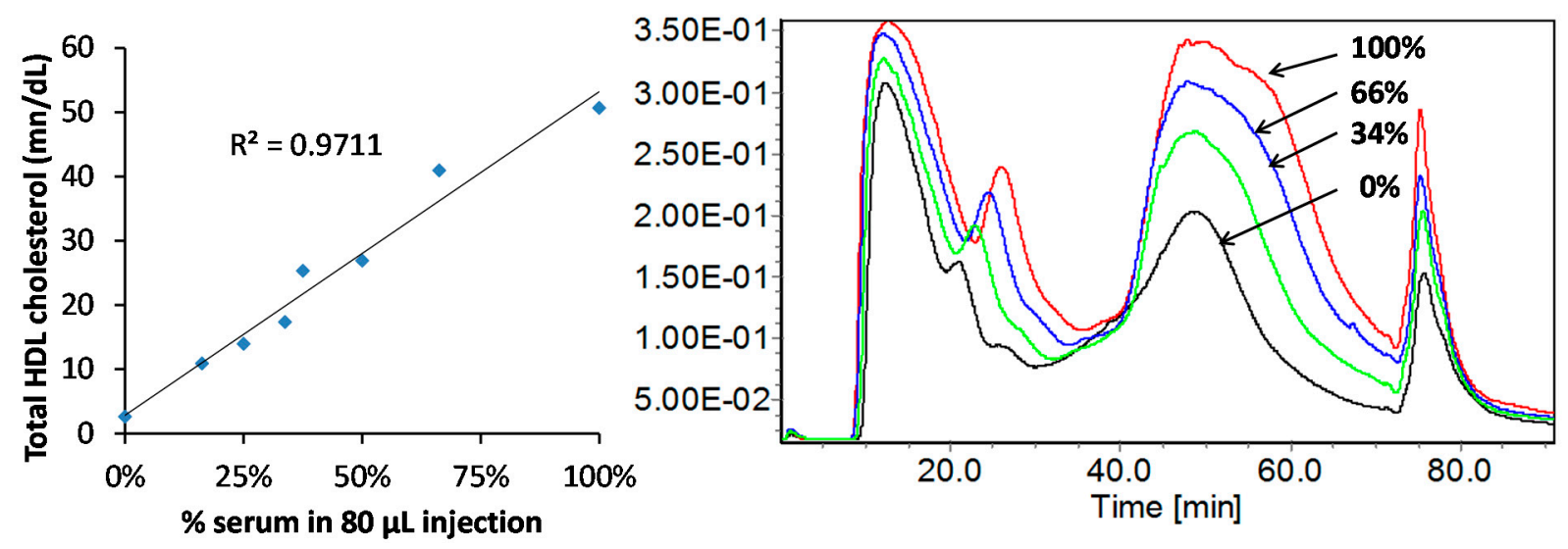

Figure 7. Dilution series of a serum sample with lipoprotein depleted serum. The total HDL cholesterol content $(\mathrm{mg} / \mathrm{dL})$ of each diluted serum was calculated from the sum of the individually measured HDL fractions as function of $\%$ non-depleted serum in $80 \mu \mathrm{L}$ injection (left). AF4 conditions: $F_{i n j}=0.2 \mathrm{~mL} / \mathrm{min}, F_{\text {cross }}=3.2 \mathrm{~mL} / \mathrm{min}, F_{\text {out }}=0.45 \mathrm{~mL} / \mathrm{min}, t_{i n j}=7 \mathrm{~min}$, $t^{\text {end }}=70 \mathrm{~min}, F_{\text {cross }}{ }^{\text {end }}=0.75 \mathrm{~mL} / \mathrm{min}$ and gradient decay function $n=0.7$.

\subsection{Analysis of Human Serum Samples}

We prepared split aliquots of serum samples from three different donors (QC1, QC2 and QC3) and analyzed them in six runs on three different days over the course of one week. The overlay of the UV fractograms showed excellent agreement in and between days (Figure 8). Depending on the DLS signal intensity and concentration in the fractions, the size determination by DLS had $\pm 1-2 \mathrm{~nm}$ accuracy. Correlating the retention times at the HSA and LDL containing peak maxima with the measured size in the corresponding fractions gave a simple two point linear calibration curve. The HDL containing peak was not included into the calibration because of its overlap with the much more intense HSA peak. The calibration curve slopes had a $7 \% \mathrm{CV}$. The linear regression equations of the calibration curves (shown in Figure 8) were used for the conversion of the retention time scale to size scale in the 7-30 nm size range of our interest.

We monitored specific target peptides for over 30 serum proteins based on LC-MS/MS signal intensity. Some proteins were present consistently in particular fractions with $\pm 1 \mathrm{~nm}$ size differences, which was indicative of the precision of the retention time to size scale conversion based on the above two point calibration procedure. As an example, the size profiles for two of these proteins (haptoglobin and transferrin) are shown in Figure 9. The $\mathrm{W}_{1 / 2}$ of these size profiles were $\sim 3 \mathrm{~nm}$, representative of near homogenous size species in serum. This also implies that HDL and LDL profiles with $\mathrm{W}_{1 / 2}>3 \mathrm{~nm}$ are the result of multiple sub-species. In future studies, we will explore how these HDL and LDL sub-classes differ in protein composition to explore cholesterol transfer dynamics based on analysis of serum samples from normal and dyslipidemic donors.

Each $80 \mu \mathrm{L}$ serum injection into the AF4 system was collected into a total of $\sim 6 \mathrm{~mL}$ elution volume. As a result of the AF4 separation, the HDL and LDL content of each serum sample was diluted $\sim 75$ fold. 
Representative LC-MS/MS chromatograms of fractions collected at HDL and LDL size profile maxima are shown in Figure 10.
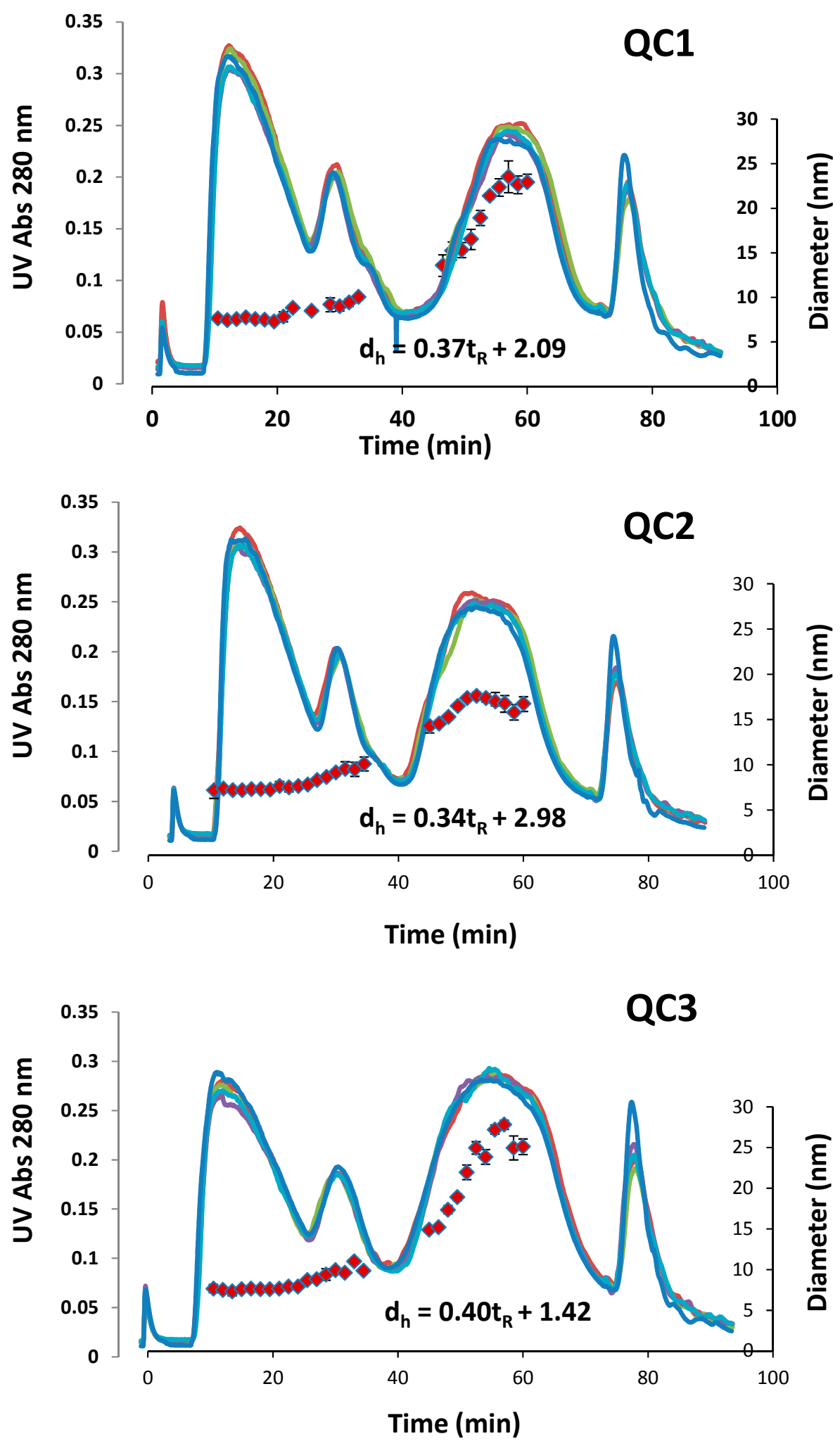

Figure 8. Overlay of UV fractograms for three serum samples from six AF4 runs from three different days over a period of one week. 


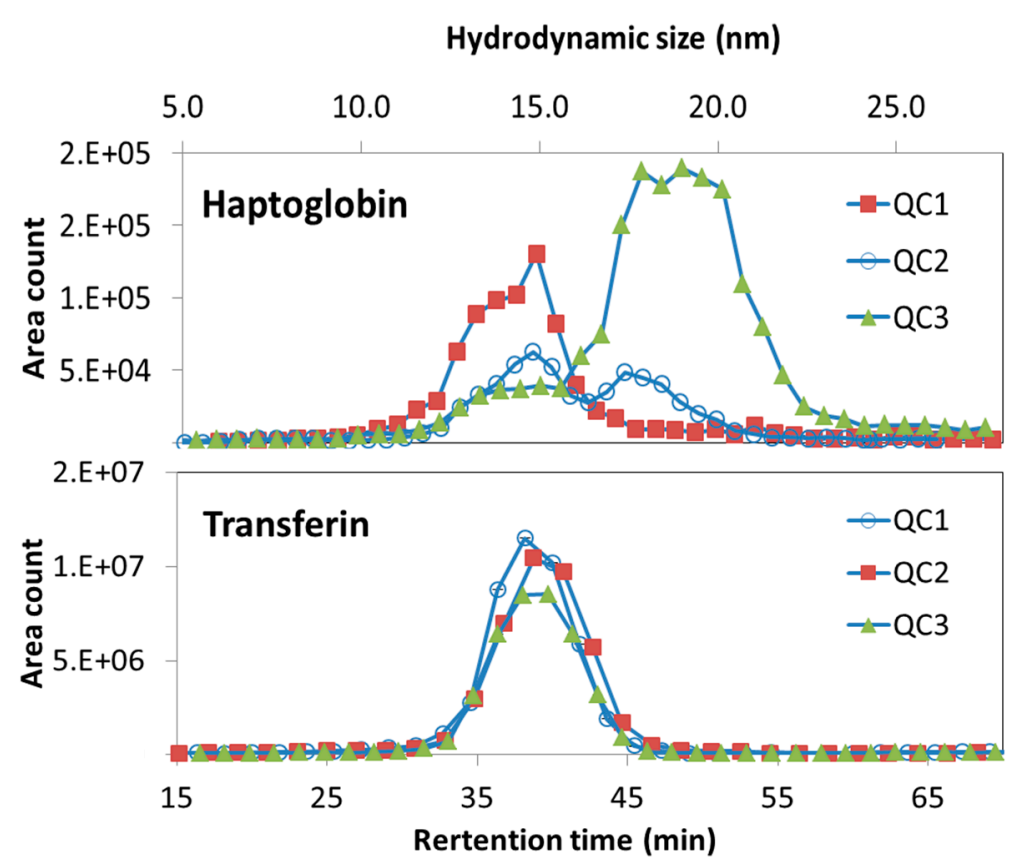

Figure 9. Average fractograms for haptoglobin and transferrin monitored by LC-MS/MS analysis of individual fractions. The retention time scale was converted to particle diameter scale based on the dynamic light scattering measurements and conversion formulas shown in overlay on the UV fractograms in Figure 8.

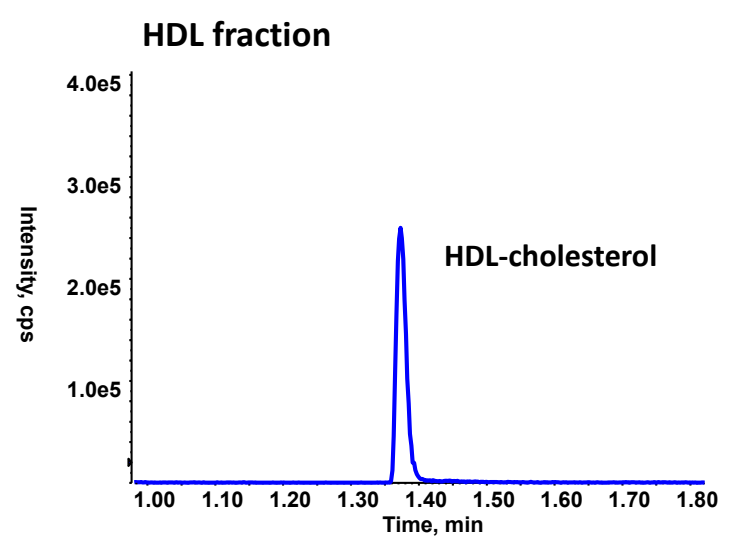

HDL fraction

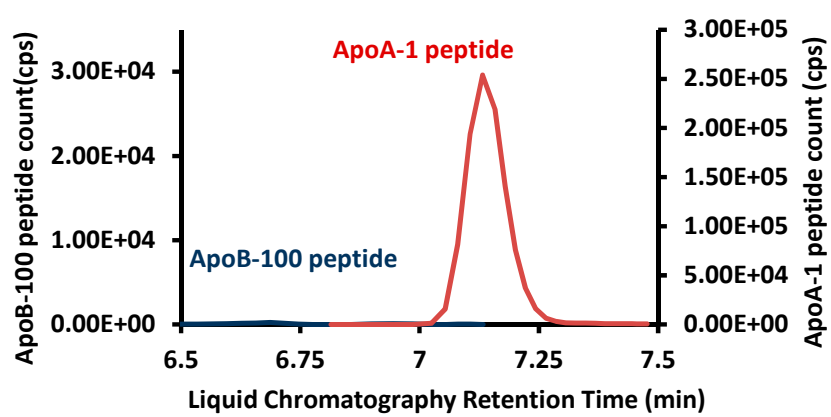

LDL fraction

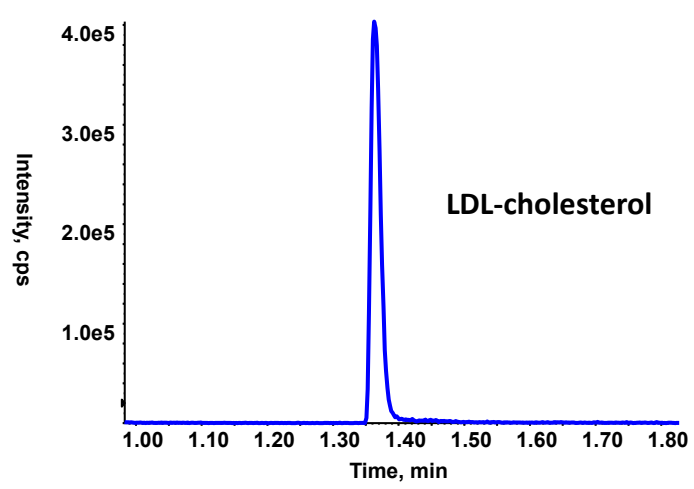

LDL fraction

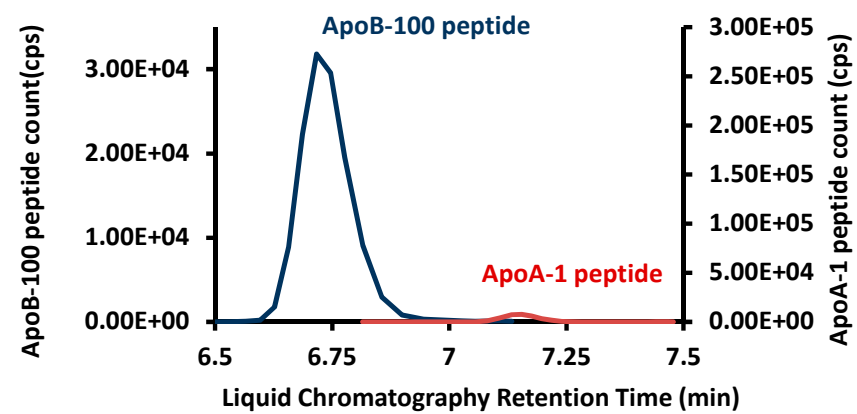

Figure 10. Typical LC-MS/MS chromatograms for fractions collected at the HDL and LDL size profile maxima for QC3, $12 \mathrm{~nm}$ and $23 \mathrm{~nm}$ fractions in Figure 11, respectively. 
Each fraction was treated as an individual sub-species of HDL and LDL in serum. The analyte amounts measured in the fractions were converted to serum concentrations according to the following formula:

$$
\text { [subspecies concentration in serum] }=\frac{\text { [analyte mass or moles in fraction] }}{\text { [volume of serum injected into the AF4 channel] }}
$$

which gave mg/dL HDL- or LDL-cholesterol concentration and nmol/L (nM) HDL- or LDL-ApoA-1 and ApoB-100 concentration for each size fraction (or sub-species) in serum (shown on each y-axis in Figure 11). Summing these fraction concentrations for cholesterol and ApoA-1 in the HDL fractions $(5-18 \mathrm{~nm})$ gave total HDL-cholesterol and total HDL-ApoA-1 concentrations in serum.

$$
\text { [total HDL subspecies concentration }]=\sum_{5 \mathrm{~nm}}^{18 \mathrm{~nm}}[\text { subspecies concentration in serum] }
$$

Similarly, summing the LDL fractions $(18-30 \mathrm{~nm})$ for cholesterol and ApoB-100 gave total LDL-cholesterol and total LDL-ApoB-100 concentration in serum:

[total LDL subspecies concentration] $=\sum_{18 \mathrm{~nm}}^{30 \mathrm{~nm}}[$ subspecies concentration in serum]
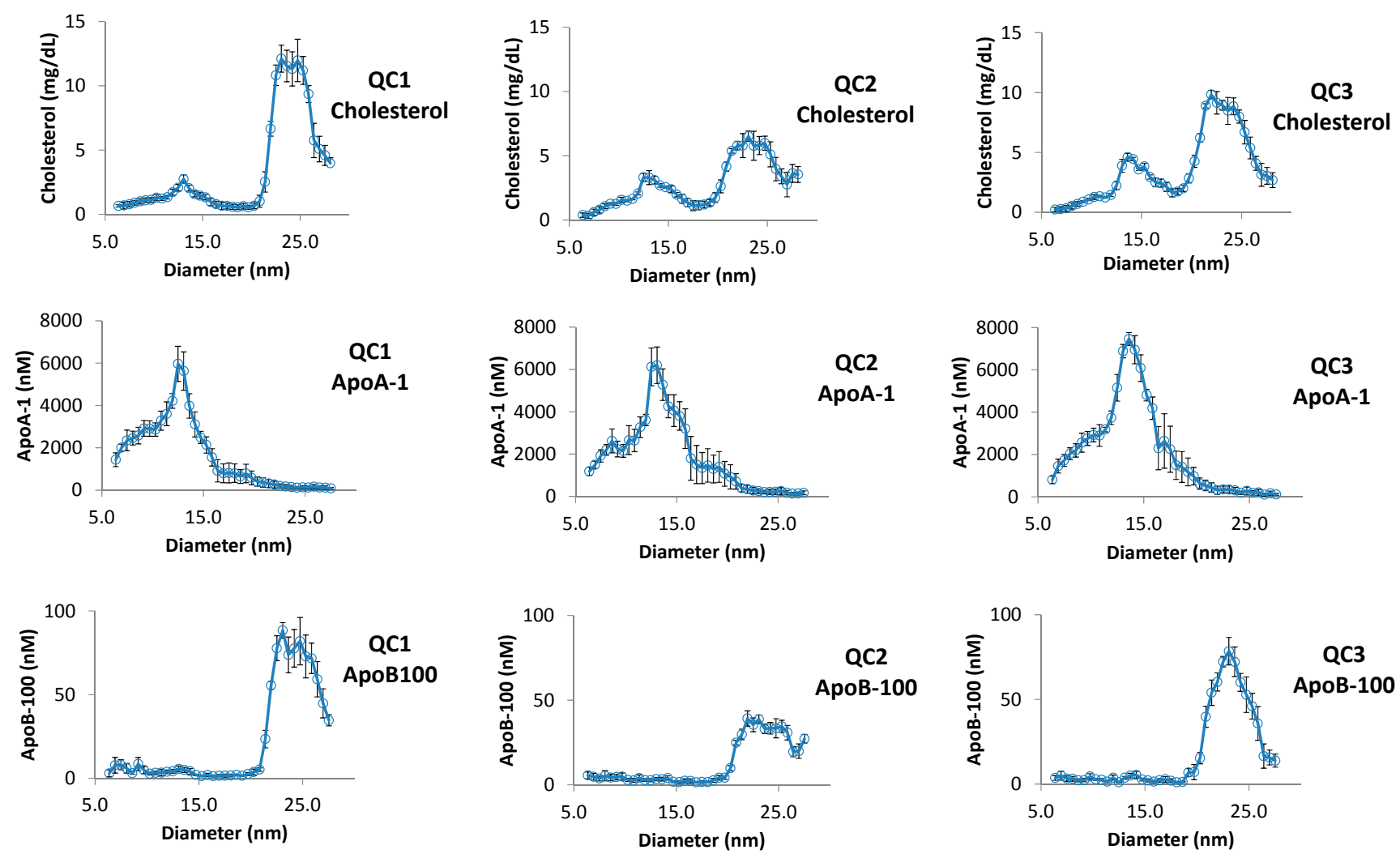

Figure 11. Inter-assay average of fractograms from repeated analysis of three serum samples collected from individual donors: cholesterol (mg/dL in serum) (top), ApoA-1 (nM in serum) (middle), and ApoB-100 (nM in serum) (bottom). The size scale was converted to diameter scale based on dynamic light scattering measurements (Figure 8). 
The total HDL and LDL subspecies concentrations in serum had $10 \%-15 \%$ inter-assay CVs. The inter-assay concentration variations in the individual size fractions were higher $(10 \%-30 \% \mathrm{CVs})$ but the profiles still show significant differences between serum samples (error bars in Figure 11).

Total channel recoveries were calculated in \% relative to the mass or moles of analyte in the volume of serum injected into the AF4 channel:

$$
\% \text { Recovery }=\frac{[\text { sum of mass or moles in the fractions }]}{\text { [total mass or moles in AF4 injection volume }]}
$$

Cholesterol and ApoB-100 are present as part of other larger lipid particles in serum such as vLDL (very low density lipoprotein) with $>60 \mathrm{~nm}$ diameter, which we did not collect. Only the HDL-cholesterol (by precipitating LDL and vLDL with $\alpha$-cyclodextrin/dextran sulfate/magnesium chloride reagent) and HDL-ApoA-1 can be measured selectively in the unfractionated serum. Therefore, the best measures of the AF4 channel recovery were the HDL-cholesterol recovery, $85 \%-90 \%$, and HDL-ApoA-1 recovery, $81 \%-87 \%$. The HDL + LDL cholesterol recovery was 68\%-75\%, and ApoB- 100 recovery was 50\%-57\%. A complete validation of the method will be presented in a later publication.

\section{Conclusions}

One of the unique power of the AF4 technique is that optimal separation can be achieved purely through adjustment of the instrumental parameters by the user, without relying on proprietary size exclusion column chromatography packing. We believe that the DoE approach empowers users to exploit the full potential of AF4 and to be able fine tune their methods with an efficient systematic manner to a specific size range and analyte group of interest, for their specific qualitative or quantitative analytical needs. Here we showed that DoE based least square fit models can be applied to predict the set of instrumental parameters that leads to optimal channel recovery while compromising the least on size resolution. We comment that performing the entire 54 run DoE on protein standards before the 15 run DoE on lipoproteins was not strictly necessary, although it was informative for demonstration of the accuracy of the theoretical DoE predictions. Our optimized method can be used for measuring particle size and quantitative stoichiometric composition of small/medium/large LDL and HDL sub-classes for the assessment of correlation with cardiovascular disease risk.

\section{Supplementary Materials}

Supplementary materials can be accessed at: http:/www.mdpi.com/2227-9075/2/1/96/s1.

\section{Acknowledgments}

This work was supported by US Federal Government funds for development of standardization of cardiovascular biomarkers. The findings and conclusions in this presentation are those of the author and do not necessarily represent the views of the Centers for Disease Control and Prevention. 


\section{Author Contributions}

ZK: project design, experimental design, MG: cholesterol analysis, BP: protein analysis, DS and JR: target peptide identification, $\mathrm{LMcW}$ : automated sample processing, YW: laboratory instrument support, JLP and JRB: Project design and guidance. All authors read and approved the final manuscript.

\section{Conflicts of Interest}

The authors declare no conflict of interest.

\section{References}

1. Giddings, J.C. Field-Flow Fractionation of Polymers: One-Phase Chromatography. Pure Appl. Chem. 1979, 51, 1459-1471.

2. Yohannes, G.; Jussila, M.; Hartonen, K.; Riekkola, M. Asymmetrical Flow Field-Flow Fractionation Technique for Separation and Characterization of Biopolymers and Bioparticles. J. Chromatogr. A 2011, 1218, 4104-4116.

3. Podzimek, S. Light Scattering, Size Exclusion Chromatography and Assymetric Flow Field Flow Fractionation: Powerful Tool for Characterization of Polumers, Proteins and Nanoparticles; Wiley: Hoboken, NJ, USA, 2011.

4. Williams, S.K.R.; Caldwell, K.D. Field-Flow Fractionation in Biopolymer Analysis; Springer: Wien, NY, USA, 2012.

5. Roda, B.; Zattoni, A.; Reschiglian, P.; Moon, M.H.; Mirasoli, M.; Michelini, E.; Roda, A. Field-Flow Fractionation in Bioanalysis: A Review of Recent Trends. Anal. Chim. Acta 2009, 635, 132-143.

6. Wahlund, K.G.; Giddings, J.C. Properties of an Asymmetrical Flow Field-Flow Fractionation Channel Having One Permeable Wall. Anal. Chem. 1987, 59, 1332-1339.

7. Wahlund, K.G. Flow Field-Flow Fractionation: Critical Overview. J. Chromatogr. A 2013, 1287, 97-112.

8. Litzen, A. Separation Speed, Retention, and Dispersion in Asymmetrical Flow Field-Flow Fractionation as Functions of Channel Dimensions and Flow-Rates. Anal. Chem. 1993, 65, 461-470.

9. Wahlund, K.G. Improved Terminology for Experimental Field-Flow Fractionation. Anal. Bioanal. Chem. 2014, 406, 1579-1583.

10. Wahlund, K.G.; Nilsson, L. Flow FFF-Basics and Key Applications. In Field-Flow Fractionation in Biopolymer Analysis; Williams, S.K.R., Caldwell, K.D., Eds.; Spinger-Verlag: Wien, NY, USA, 2012; pp. 1-21.

11. Zattoni, A.; Rambaldi, D.C.; Roda, B.; Parisi, D.; Roda, A.; Moon, M.H.; Reschiglian, P. HollowFiber Flow Field-Flow Fractionation of Whole Blood Serum. J. Chromatogr. A 2008, 1183, 135-142.

12. Reschiglian, P.; Moon, M.H. Flow Field-Flow Fractionation: A Pre-Analytical Method for Proteomics. J. Proteomics 2008, 71, 265-276.

13. Yang, I.; Kim, K.H.; Lee, J.Y.; Moon, M.H. On-Line Miniaturized Asymmetrical Flow Field-Flow Fractionation-Electrospray Ionization-Tandem Mass Spectrometry with Selected Reaction Monitoring for Quantitative Analysis of Phospholipids in Plasma Lipoproteins. J. Chromatogr. A 2014, 1324, 224-230. 
14. Kim, K.H.; Moon, M.H. Chip-Type Asymmetrical Flow Field-Flow Fractionation Channel Coupled with Mass Spectrometry for Top-Down Protein Identification. Anal. Chem. 2011, 83, 8652-8658.

15. Oh, S.; Kang, D.; Ahn, S.M.; Simpson, R.J.; Lee, B.H.; Moon, M.H. Miniaturized Asymmetrical Flow Field-Flow Fractionation: Application to Biological Vesicles. J. Sep. Sci. 2007, 30, 1082-1087.

16. Qureshi, R.N.; Kok, W.T.; Schoenmakers, P.J. Fractionation of Human Serum Lipoproteins and Simultaneous Enzymatic Determination of Cholesterol and Triglycerides. Anal. Chim. Acta 2009, 654, 85-91.

17. Park, I.; Paeng, K.J.; Yoon, Y.; Song, J.H.; Moon, M.H. Separation and Selective Detection of Lipoprotein Particles of Patients with Coronary Artery Disease by Frit-Inlet Asymmetrical Flow Field-Flow Fractionation. J. Chromatogr. B 2002, 780, 415-422.

18. Lee, J.Y.; Choi, D.; Johan, C.; Moon, M.H. Improvement of Lipoprotein Separation with a Guard Channel Prior to Asymmetrical Flow Field-Flow Fractionation Using Fluorescence Detection. J. Chromatogr. A 2011, 1218, 4144-4148.

19. Woolfitt, A.R.; Solano, M.I.; Williams, T.L.; Pirkle, J.L.; Barr, J.R. Amino Acid Analysis of Peptides Using Isobaric-Tagged Isotope Dilution Lc-Ms/Ms. Anal. Chem. 2009, 81, 3979-3985.

20. Litzen, A.; Wahlund, K.G. Zone Broadening and Dilution in Rectangular and Trapezoidal Asymmetrical Flow Field-Flow Fractionation Channels. Anal. Chem. 1991, 63, 1001-1007.

21. Giddings, J.C. Field-Flow Fractionation of Macromolecules. J. Chromatogr. A 1989, 470, 327-335.

(C) 2015 by the authors; licensee MDPI, Basel, Switzerland. This article is an open access article distributed under the terms and conditions of the Creative Commons Attribution license (http://creativecommons.org/licenses/by/4.0/). 